\title{
Fetal Spinal Cord Transplants Support the Development of Target Reaching and Coordinated Postural Adjustments after Neonatal Cervical Spinal Cord Injury
}

\author{
Pamela S. Diener and Barbara S. Bregman \\ Department of Cell Biology, Division of Neurobiology, Georgetown University Medical Center, Washington, D.C. 20007
}

Neonatal midthoracic spinal cord injury disrupts the development of postural reflexes and hindlimb locomotion. The recovery of rhythmical alternating movements, such as locomotion, is enhanced in injured animals receiving fetal spinal cord transplants. Neonatal cervical spinal cord injury disrupts not only locomotion but also skilled forelimb movement. The aims of this study were to determine the consequences of cervical spinal cord injury on forelimb motor function and to determine whether transplants of fetal spinal cord support normal development of skilled forelimb use after this injury. Three-day-old rats received a cervical spinal cord lesion at C3, with or without a transplant of fetal cervical spinal cord (embryonic day 14); unoperated pups served as controls. Animals were examined daily during the first month of life using a behavioral protocol that assessed reflexes, postural reactions, and forelimb motor skills. They also were trained and tested as adults to assess performance in goal-directed reaching tasks. The onset of pos- tural reflexes was delayed in the lesion-only group, and goaldirected reaching and associated postural adjustments failed to develop. The transplant group developed reflex responses and skilled forelimb activity that resembled normal movement patterns. Transplant animals developed both target reaching and accompanying postural adjustments. Target reaching requires integration of segmental, intersegmental, and supraspinal input to propriospinal and motor neurons over many spinal cord levels. Transplants may support the reestablishment of input onto these neurons, permitting the development of skilled forelimb activity after neonatal cervical spinal cord injury. The neuroanatomical reorganization of descending and propriospinal input was examined in the companion paper (Diener and Bregman, 1998).

Key words: recovery of function; neonatal rat; reaching; postural adjustments; transplants; development; behavior
The development of hindlimb locomotion and the coordination between forelimbs and hindlimbs relies on the formation of interconnections between supraspinal, propriospinal, and segmental axons, with local pattern generators for each limb (Grillner, 1973, 1975, 1976, 1986; Andersson et al., 1978). Midthoracic spinal cord injury damages the input to hindlimb pattern generators and disrupts reflex development and normal hindlimb movements for locomotion. The consequences of cervical spinal cord injury are more severe than those after midthoracic injury. There are, for example, greater temporal delays in the development of reflexes and locomotion, and more abnormal movement patterns persist after cervical injury than after thoracic injury (Bregman and Goldberger, 1983). Forelimb deficits are also pronounced in various aspects of motor function after high cervical spinal cord injury in the adult (Schrimsher and Reier, 1992, 1993). After

\footnotetext{
Received June 26, 1997; revised Oct. 22, 1997; accepted Oct. 27, 1997.

This work was supported by National Institutes of Health Grants NS27054 and NS19259 from the National Institute of Neurological Disorders and Stroke and Grant T32 HD07459 from the National Center for Medical Rehabilitation Research to B.S.B. The behavioral and anatomical results represented in this and the companion paper describe many of the studies detailed in the doctoral dissertation by P.S.D. (1995). We are grateful to Dr. Gary Chase for assistance in statistical analysis, Dr. Ronald L. Diener for assistance with equipment fabrication, and Hai-Ning Dai, Cara James, and Marietta McAtee for surgical and technical assistance. We thank Dr. Holli Bernstein-Goral, Dr. Melinda Kelley, and Marietta McAtee for their helpful suggestions on this manuscript.

Correspondence should be addressed to Dr. Barbara S. Bregman, Department of Cell Biology, Georgetown University Medical Center, 3900 Reservoir Road NW, Washington, D.C. 20007.

Dr. Diener's present address: Department of Physical Therapy, Marymount University, 2807 N. Glebe Road, Arlington, VA 22207-4299.

Copyright (C) 1998 Society for Neuroscience $\quad 0270-6474 / 98 / 180763-16 \$ 05.00 / 0$
}

spinal cord injury in the adult rat, for example, movement required for reaching toward a target placed on the body recovers, whereas movements required to reach for targets placed away from the body fail to recover. To date, little is known about (1) the development and maturation of skilled forelimb activity such as goal-directed reaching and (2) the development of the postural control that is essential for precise forelimb movements.

It is well established that skilled activity involves a more complex integrative neural network than that interacting with local pattern generators for locomotion (Grillner, 1975, 1976; Andersson et al., 1978; Alstermark et al., 1984a,b,c, 1987c, 1990, 1991a). The development of reaching after neonatal spinal cord injury, therefore, may not be as extensive as the recovery of hindlimb locomotion. The first aim of this study was to examine the development and maturation of forelimb reaching and to determine the effect of upper cervical spinal cord injury on this development. The second aim of this study was to determine whether transplants have the capacity to mediate recovery of skilled forelimb movements such as reaching or whether recovery is restricted to rhythmic alternating movements such as locomotion.

It is clear that transplants of fetal spinal cord tissue enhance the development of rhythmical alternating movements after neonatal midthoracic spinal cord injury (Kunkel-Bagden and Bregman, 1990; Kunkel-Bagden et al., 1992; Bregman et al., 1993; Howland et al., 1995). Transplants support the survival of immature axotomized neurons and the regrowth of axons to spinal cord levels caudal to the injury (Bregman and Reier, 1986; Bregman, 1987a,b; Bernstein-Goral and Bregman, 1993; Diener and Breg- 
man, 1994). This axonal regrowth is associated with improved locomotor function in animals with transplants compared with permanent deficits in locomotion in the lesion-only animals. The effects of transplantation on the development of skilled, goaldirected forelimb activity after neonatal cervical spinal cord injury, however, are unknown. To assess the contribution of the transplant to the development of reaching, a behavioral testing battery was designed to evaluate the execution of motor skills required for reaching in developing animals and in mature animals that had been injured at birth. The results indicate that marked developmental delays and abnormal compensatory motor patterns emerge in lesion-only animals and reaching fails to develop. In contrast, skilled forelimb activity develops and mature reaching for targets on and away from the body is established in both transplant and control animals. The greater recovery of skilled forelimb movement is associated with greater supraspinal input to the spinal cord in the presence of the transplants (see companion paper, Diener and Bregman, 1998).

\section{MATERIALS AND METHODS}

\section{Cervical spinal cord injury and transplantation}

Timed-pregnant Sprague Dawley rats were obtained from Zivic Miller Laboratories (Zelienople, PA) and observed two times daily for the presence of pups. Day of birth was designated postnatal day (P) 1. Eighty-four newborn rats were prepared for this experiment. The rats were divided randomly into three groups: control (CON, $n=13)$, overhemisection only ( $\mathrm{HX}, n=33$ ), and overhemisection plus fetal transplant $(\mathrm{HX}+\mathrm{TP}, n=38)$. On the third postnatal day, operate pups were anesthetized by hypothermia. An incision was made along the skin overlying the cervical vertebrae. The muscle fibers were separated to expose the underlying vertebral column. A laminectomy of the $\mathrm{C} 2$ and $\mathrm{C} 3$ segments was performed, and the dura overlying these segments was slit longitudinally with a no. 11 surgical blade. The right side of the $\mathrm{C} 3$ spinal cord segment was lesioned (overhemisection) using iridectomy scissors. An overhemisection of the spinal cord destroys the dorsal funiculus bilaterally and the right ventral and lateral funiculi and the intervening gray matter unilaterally. In a separate group of animals (HX $+\mathrm{TP})$, after the spinal cord was lesioned, one or two pieces of embryonic cervical spinal cord $\left(1-2 \mathrm{~mm}^{2}\right)$ were inserted into the cavity created by the retraction of the cut ends of the cord. The donor tissue was obtained from anesthetized (chloral hydrate, $400 \mathrm{mg} / \mathrm{kg}$, i.p.) timed-pregnant Sprague Dawley rats $14 \mathrm{~d}$ in gestation (E14) and dissected under aseptic conditions in DMEM as described previously (Reier et al., 1986; Bregman and McAtee, 1993). After lesion or lesion plus transplantation, the injury site was covered with synthetic dura (durafilm, Codman-Schurtleff, Inc.) and covered with $0.9 \%$ saline-soaked gelfoam. The overlying muscle and skin were sutured with 6.0 silk. The pups recovered in a warm environment and were injected with a prophylactic dose of bicillin (Wyeth Laboratories, Philadelphia, PA) subcutaneously and returned to their mothers.

\section{Behavioral testing}

Rats were tested daily from birth through P30 for the developmental series. Rats also were trained and tested as adults ( $>4$ weeks) on various reaching and other motor tasks. The stand $(109 \times 37.5 \times 106 \mathrm{~cm})$ used for behavioral testing was constructed with a glass top measuring $44 \times 109 \mathrm{~cm}$ and an angled mirror $(34 \times 125 \mathrm{~cm})$ to permit simultaneous viewing of the lateral and ventral aspects of the rat. Developing and mature rats were assessed qualitatively and quantitatively for forelimb use and associated body adjustments. Scoring criteria based on normal components of each particular motor activity were established for each test.

Developmental and postural reflexes that were examined included righting, chin placing, grasp reflex, and motor response to noxious stimuli. Postural reflex testing examined both full body and individual forelimb movements, whereas individual limb reflexes analyzed specific components of limb function. Additionally, other forelimb skills were used to study postural reactions, proximal control and stability, the use of the forelimb for goal-directed movements, coordinated bilateral forelimb movements, and distal control (tested by grasping). The use of accurate forelimb aiming during the rhythmical alternating movement of locomo- tion was evaluated qualitatively and quantitatively. At the end of the 4 weeks of testing, randomly selected rats from each group (CON, $n=6$; HX, $n=3$; HX + TP, $n=3$ ) were perfused, and the spinal cords and brains were dissected. This tissue was prepared for analysis of descending and segmental projections that may remodel after injury to the developing spinal cord and serve as the underlying mechanism(s) for the observed motor behaviors (Diener and Bregman, 1998). At the sixth through ninth week of life, the remaining rats in each group were trained and tested for forelimb use in target-directed reaching. Rats in all groups were trained to reach at midline to remove a sticker from their heads (simulating a grooming response) and to reach forward (grasp and retrieve a food pellet) away from their bodies to challenge further their balance reactions. For each test, the training period lasted 1 week, followed by 1 week of testing.

\section{Videotape recordings}

Behavioral testing for each group was recorded on videotape approximately once a week. The animals were filmed with a Panasonic camera (WV-3260/8F) with a shutter speed of $1 / 1000 \mathrm{sec}$. When possible, mirrors were incorporated to allow simultaneous viewing of the rat from lateral and ventral planes. For target reaching only, the performance was recorded from both a lateral and anterior view. Analysis of the videotapes occurred after all testing was completed. To analyze the component movements used by the developing and adult rats, the videotapes were viewed with good resolution in slow motion.

\section{Developmental tests and scoring criteria}

There are a number of motor skills that are prerequisite for the development of target-directed reaching. These include (in sequence) wholebody control, proximal stabilization for distal mobility, and anticipatory postural adjustments for reaching. Scoring criteria for individual assessments are summarized in Table 1 .

Righting. The righting reflex develops postnatally (Kunkel-Bagden et al., 1992). Righting the body requires sequential upper and lower body movements to turn from supine to prone. Because righting requires the use of the forelimbs and the upper body, this reflex was assessed to determine whether (1) high spinal cord injury would delay the date of reflex development, (2) forelimb use would be compromised, and (3) aberrant behaviors would develop. The ability to right was tested by placing and holding the rat still in a supine position on the glass table top. The time to turn from supine to prone was measured with a stopwatch and recorded in seconds. The characteristics of the movement were described according to a five-point scale (Table 1).

Spatial orientation. Orientation of the body in space was measured by using a board inclined to a $30^{\circ}$ angle. Both the time to orient the body to face upward and the motor patterns used to turn the body around were recorded. The rat was placed with all paws on the board, with its body and nose facing down. A five-point rating scale (Table 1) was used to evaluate the motor components used by the rat to achieve the upright posture.

Chin placing. The stimulus of placing the chin on a surface is another reflex that incorporates bilateral forelimb use in the response. To achieve light pressure on the ventral surface of the chin, the examiner gently supported the forelimbs and lower trunk/hindlimbs and rested the rat's chin on a smooth, horizontal surface. The timer was started as the examiner released the support on the forelimbs. The number of seconds required by the rat to place both paws on the surface and the exact sequence of paw placement were recorded for each of three trials. Because the eyes are still closed at P11-12, chin placing is not influenced by visual input. The rats were rated according to a three-point scale for limb placement (Table 1).

Grasping. Before active grasping and proximal control were tested, each rat was evaluated to determine whether it had either an immature grasp reflex or a volitional active grasp. The stimulus for active grasping, a 15 -cm-long wooden rod ( $2 \mathrm{~mm}$ in diameter) was applied to the volar surface of the forepaw of newborn rats. Strong wrapping of the digits around the rod as it was brushed over the palm combined with the inability to release the rod by extending the digits was recorded as an immature grasp reflex. If the rat could grasp and release its grip on the rod, the grasp was recorded as volitional. To assess the ability of the rat to grasp and maintain proximal control, the rats were evaluated as they held onto the suspended rod. The rod was raised above the surface to prevent the body/tail from contacting the table top and therefore from assisting with postural support. The number of seconds that the rat held onto the rod was recorded. Descriptions of forelimb use were docu- 


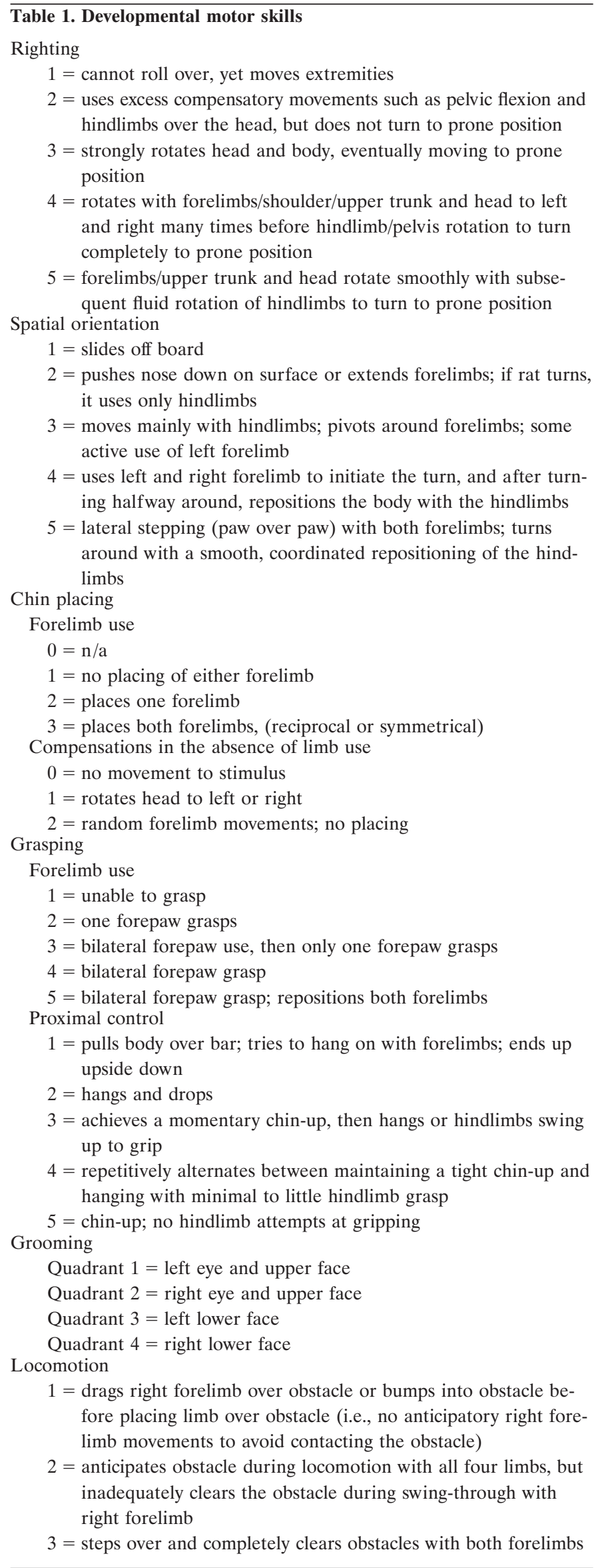

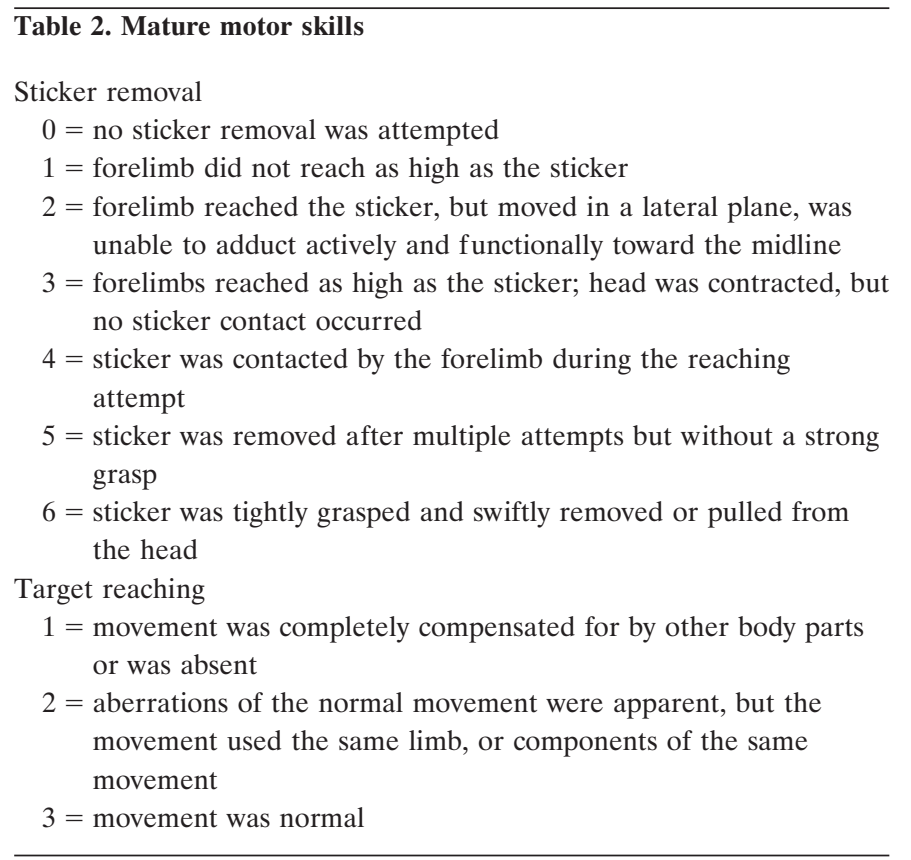

mented using two separate five-point scales, one to evaluate forelimb use and a second to evaluate proximal limb control (Table 1).

Grooming. Functional use of the forelimbs was evaluated first by examining the active range of motion of each forelimb. To assess full active forelimb use in each rat, pups were placed on the glass top of the stand, and a noxious stimulus (cold water) was applied to the face of each rat. Each rat's forelimb and body responses were recorded on videotape (viewing lateral and ventral sides of the animal). The development of bilateral forelimb range of motion in response to the stimulus was described qualitatively and quantitatively. Each rat's face was divided into four quadrants: a vertical line was drawn along the midline of the face, and a horizontal line was drawn just caudal to the eyes to comprise the four quadrants. The animals were rated to determine the functional range of motion for each forelimb by recording the quadrant to which the forelimb reached (Table 1) and the number of times it entered that quadrant.

Locomotion. The ability of a rat to step over obstacles during locomotion was examined after overground locomotion developed fully. Stepping over an obstacle requires anticipatory commands both to adjust postural support and to initiate sufficient limb flexion to clear the obstacle (Drew, 1991). A short runway $(10 \times 42.5 \mathrm{~cm})$ with 1.25 -cm-high obstacles placed $11.25 \mathrm{~cm}$ apart was used for testing. The rat was placed at one end and walked from one end to the other during a 2-3 min trial; its ability to step over the obstacle was scored using a three-point scale (Table 1).

Adult tests and scoring criteria. Forelimb motor skills and postural reactions were also assessed after the rats reached maturity (at least 4 weeks of age). Scoring criteria for individual assessments are summarized in Table 2.

Sticker removal. Removal of a sticker placed on the bridge of a rat's nose required full range of motion of the forelimbs and at least some grasping ability. Assessment began with placement of a sticker $(1.9 \mathrm{~cm}$ in diameter) on the rat's head between the nose and eyes. After a training period of 1-2 d, the number of times and the extent to which each forelimb reached to remove the sticker were recorded. A rat was given a maximum of $2 \mathrm{~min}$ to remove each sticker (three stickers per trial, three trials per animal). The scoring measures were similar to those used by Schrimsher and Reier (1992). Scoring was calculated individually for each forelimb (Table 2).

Adult target reaching. Forward reaching involves both forelimb use and associated postural adjustments. Goal-directed reaching into a specified region for a food pellet (target) was analyzed in adult rats after a $48 \mathrm{hr}$ fast from a solid diet. The design of the reaching apparatus was a modification of those used in other laboratories (Whishaw and Kolb, 1988; Schrimsher and Reier, 1992, 1993). The reaching apparatus was constructed with a metal grate $(1.25 \mathrm{~cm}$ between vertical rods) as the front wall and Plexiglas for the remaining walls to make a box $30 \times$ 
$15.6 \times 20 \mathrm{~cm}$. The box contained a series of 12 cubbies/shelves $(2.5 \times$ $2.5 \times 2.5 \mathrm{~cm})$ situated in four rows and three columns with four open shelves $(2.5 \times 2.5 \mathrm{~cm})$ above. A space of $0.6 \mathrm{~cm}$ was maintained between the shelves and the grate wall. This separation between the cubby and the grate wall ensured that the pellet could not be dragged from the shelf and shuttled into the mouth. Instead, if the grasp was inaccurate and the pellet fell as the limb was withdrawn from the cubby, the rat could not retrieve it. Similarly, the floor of the apparatus was a grate with holes of 0.6 by $0.6 \mathrm{~cm}$. If the pellet was dropped as the limb approached the mouth, the pellet would fall through the grate and be unobtainable. In this way, being rewarded by the food was possible only if the rat accurately maintained its grasp around the pellet. Rats were weighed daily to verify that their weight never dropped below $80 \%$ of baseline. Water was provided ad libitum. After the $48 \mathrm{hr}$ fast, rats received $45 \mathrm{mg}$ food pellets (P.J. Noyes Company, Lancaster, NH) during a $5 \mathrm{~min}$ trial. If they reached for the food pellets during the training period, they were rewarded with their regular diet for 15-20 min; all rats received a portion of their regular diet overnight. Testing sessions occurred after the 1 week training period, which prepared the rats to reach into the horizontal shelves to grasp and eat the $45 \mathrm{mg}$ food pellets. At the end of each test session, all rats received their regular diet for 15-20 min.

Scoring of forelimb use was accomplished in several ways. Rats reached into a total of 16 shelves set at four different heights as described above. Each shelf contained four $45 \mathrm{mg}$ food pellets to total 64 pellets at the beginning of the $5 \mathrm{~min}$ trial. Each daily session documented both the number of pellets that were dropped onto the surface and those that remained in the cubby. The total was then subtracted from 64 to determine the number of pellets consumed. Components of reaching and postural adjustments were rated using a scale (Table 2) modified from that described by Whishaw and colleagues (Miklyaeva et al., 1994).

The components of forelimb reaching that were evaluated included raising the forelimb from its weight-supporting posture, neutral positioning of the forelimb in midline with digit flexion, forward reaching into a cubby with the forelimb moving along the midline, extending the forelimb over the pellet that was situated in the cubby, extending the digits over the pellet, pronating the forelimb to prepare for the grasp, flexing the digits and grasping the pellet, lifting and slightly supinating the forelimb to withdraw the limb, bringing the forepaw to midline under the mouth, and finally bringing the opposite forelimb to midline to assist in holding the pellet while eating. The component parts of the postural reactions were scored by the same three-point scale. The individual motor components assessed included recording the position of the nonreaching forelimb (e.g., remained on the ground to support the body weight), the quality of the reach (e.g., precise or multiple random attempts vs substitution of other body parts), the method of grasping and withdrawing the forelimb (e.g., grasping pellet, paw inspection before or after pellet dropped), the lower body and hindlimb base of support (e.g. use of a stable base vs continual repositioning to maintain balance vs losing balance), and the coordination between the forelimbs and hindlimbs.

\section{Lesion reconstruction}

At 4 weeks to 4 months after cervical spinal cord injury with or without transplantation, randomly selected rats were overdosed (chloral hydrate, $1000 \mathrm{mg} / \mathrm{kg}$, i.p.) and perfused intracardially with $0.9 \%$ heparinized saline followed by $4 \%$ paraformaldehyde in a $0.1 \mathrm{M}$ phosphate buffer, $\mathrm{pH}$ 7.4. Directly after perfusions, the spinal cord and brain were removed and post-fixed at room temperature for $2 \mathrm{hr}$. Tissue was cryoprotected in a graded series of sucrose solutions $(10-30 \%)$ at $4^{\circ} \mathrm{C}$. Spinal cord tissue was blocked and cut in cross sections on a cryostat (16 $\mu \mathrm{m}$ sections), and a 1:5 series was mounted on subbed slides. One slide from each series was stained with cresyl violet to document the extent of the lesion and distinguish transplant tissue from host spinal cord. The lesion and transplant sites were serially reconstructed using an aus Jena microprojector to determine the rostrocaudal and transverse extent of the lesion site and transplant apposition. Strict lesion criteria were established to ensure uniformity among animals used in the study. All rats included in the behavioral analysis had a lesion that ablated the dorsal columns bilaterally and the lateral and ventral funiculi and intervening gray matter unilaterally (right side). Consequently, descending pathways contributing to the development of right forelimb use were axotomized. Inclusion in the $\mathrm{HX}+\mathrm{TP}$ group also required extensive rostrocaudal and transverse apposition of the transplant to the host tissue. Table 3 lists the animals that met all of the criteria for inclusion in the final data analysis and indicates the behavioral tests conducted on each. All of these animals used in the behavioral studies also underwent anatomical analysis to determine the extent of reorganization of supraspinal and propriospinal neurons after cervical spinal cord lesions and transplants (Diener and Bregman, 1998).

\section{Statistics}

The quantitative results of behavioral tests from each group were compared using the SPSS (Statistical Program for Social Sciences, Chicago, IL) computer program. All results are expressed as the mean $\pm \mathrm{SD}$. The rating scores of each behavioral test were ordered scales and served as the dependent variables in the analysis against the animal groups, which were defined by surgical procedure (independent variable). Differences were compared across all groups using one-way ANOVA. If variability existed among the groups, specific differences were determined by comparing the control group and each experimental group (CON vs HX, CON vs $\mathrm{HX}+$ $\mathrm{TP}$ ) or the experimental groups against each other (HX vs HX + TP). These between-group analyses were performed using the Bonferroni, Scheffé, and Tukey tests for significance, with a $p$ value of 0.05 .

\section{RESULTS}

\section{Lesion reconstructions}

Representative cross sections from the lesion site in hemisection and hemisection plus transplant rats included in the behavioral analysis are illustrated in Figure $1, A$ and $B$, respectively. The motor behavior observed within each group of animals was generally consistent. For example, of the animals that met the lesion criteria for inclusion in the study, in each treatment group the motor behavior of animals with the smallest acceptable transverse lesion and the largest acceptable transverse lesions was similar qualitatively and quantitatively. Thus, differences observed in the motor performance between lesion-only and lesion plus transplant animals on specific tasks could not be attributed to differences in the transverse extent of the injury. The experimental animals described in this section survived the surgery and completed all behavioral testing and analysis. Table 3 lists the animals in each group that met all lesion criteria and indicates which behavioral analysis was performed on which animals. In each group, rats were withheld from behavioral analysis if they survived the initial surgery but did not survive beyond 10-14 d (HX, $n=7 ; \mathrm{HX}+\mathrm{TP}, n=10)$, did not meet the lesion criteria (HX, $n=2 ; \mathrm{HX}+\mathrm{TP}, n=5$ ), or for unknown reasons (unrelated to the injury incurred) failed to train in response to food reward $(\mathrm{HX}, n=3)$. The results reported below are based on final sample sizes of CON, $n=13$; HX, $n=10$; and HX + TP, $n=8$.

\section{Development of postural reflexes and forelimb movement: whole-body responses to sensory stimulation \\ Righting}

The body-righting reflex matured by $\mathrm{P} 10.6 \pm 1.2$ in normal rats (Fig. 2A,CON). Normal rats exhibited a similar motor sequence as they developed righting responses. The supine position initially elicited random movements of all four extremities, with exaggerated flexion of the neck and abdomen. As the response matured, there was a reduction in the time to right, and pelvic and upper body rotation became isolated. The upper body rotated first in synchrony with the forelimbs, and then the lower body rotated and hindpaws were placed under the body (Fig. $2 B$; rating scale $=5$ ).

Cervical spinal cord hemisection delayed the development and maturation of body righting. The time to right was increased, and abnormal movement patterns persisted. For example, in response to being placed supine, an exaggerated version of the normal immature response was used and persisted for several weeks. Lesion-only rats failed to develop isolated upper and lower body rotation. Instead, they used extreme neck rotation or excessive 
Table 3. Behavioral tests of forelimb use during development and at maturity

\begin{tabular}{|c|c|c|c|c|c|c|c|c|c|c|}
\hline \multirow[b]{2}{*}{ litters } & \multicolumn{6}{|c|}{ forelimb use during development } & \multicolumn{3}{|c|}{ forelimb use during maturity } & \multirow[b]{2}{*}{ Anatomy: } \\
\hline & righting & orient & grasp] & chin & stepping & grooming & reaching & postural & sticker & \\
\hline & & & & placing & over & & & adjustments & removal & sc cross sxns \\
\hline \multicolumn{11}{|l|}{$\mathrm{CON}$} \\
\hline $\mathrm{CON}-1$ & $x$ & $\mathrm{x}$ & $\mathrm{x}$ & $\mathrm{x}$ & $\mathrm{x}$ & $\mathrm{x}$ & & & & normal \\
\hline CON-2 & $x$ & $\mathrm{x}$ & $x$ & $x$ & $x$ & $x$ & $\mathrm{x}$ & $\mathrm{x}$ & $\mathrm{x}$ & normal \\
\hline $\mathrm{CON}-3$ & $x$ & $x$ & $x$ & $x$ & $x$ & $x$ & $x$ & $x$ & $x$ & normal \\
\hline $\mathrm{CON}-4$ & & & & & & & $x$ & $\mathrm{x}$ & $\mathrm{x}$ & normal \\
\hline CON-5 & & & & & & & $x$ & $x$ & $x$ & normal \\
\hline CON-6 & & & $\mathbf{x}$ & $\mathrm{x}$ & & & & & & normal \\
\hline CON-7 & & & $x$ & $x$ & & & & & & normal \\
\hline CON-8 & & & $x$ & $x$ & & & & & & normal \\
\hline CON-9 & & & $x$ & $x$ & & & & & & normal \\
\hline CON-10 & & & & $x$ & & & & & & normal \\
\hline $\mathrm{CON}-11$ & & & $x$ & $x$ & & & & & & normal \\
\hline \multicolumn{11}{|l|}{$\mathrm{HX}$} \\
\hline$H X-1$ & $x$ & $x$ & $x$ & $x$ & & $x$ & $x$ & $x$ & $x$ & 30 \\
\hline $\mathrm{HX}-2$ & $x$ & $x$ & $x$ & $x$ & $x$ & $x$ & $x$ & $x$ & $x$ & $\Leftrightarrow$ \\
\hline $\mathrm{HX}-3$ & $x$ & $x$ & $x$ & $x$ & $x$ & $x$ & & & & 3 \\
\hline $\mathrm{HX}-4$ & $x$ & $x$ & $x$ & $x$ & $x$ & $x$ & $x$ & $x$ & $x$ & (2) \\
\hline $\mathrm{HX}-5$ & & & & & & & $x$ & $x$ & $x$ & 30 \\
\hline HX-6 & & & & & & & $x$ & $x$ & $x$ & \\
\hline $\mathrm{HX}-7$ & $x$ & $x$ & $x$ & $x$ & & & & & & 3 \\
\hline$H X-8$ & $x$ & $x$ & $x$ & $x$ & & & & & & 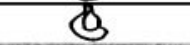 \\
\hline \multicolumn{11}{|l|}{$\mathrm{HX}+\mathrm{TP}$} \\
\hline $\mathrm{HX}+\mathrm{TP}-1$ & $x$ & $x$ & $x$ & $x$ & $x$ & $x$ & $x$ & $x$ & $x$ & (2) \\
\hline $\mathrm{HX}+\mathrm{TP}-2$ & $x$ & $x$ & $x$ & $x$ & $x$ & $x$ & $x$ & $x$ & $x$ & 20 \\
\hline HX+TP-3 & $x$ & $x$ & $x$ & $x$ & $x$ & $x$ & $x$ & $x$ & $x$ & (C) \\
\hline $\mathrm{HX}+\mathrm{TP}-4$ & $x$ & $x$ & $x$ & $x$ & $x$ & $x$ & $x$ & $x$ & $x$ & टर्रि \\
\hline HX+TP-5 & $x$ & $x$ & $x$ & $x$ & $x$ & $x$ & $x$ & $x$ & $x$ & (2) \\
\hline HX+TP-6 & $x$ & $x$ & $x$ & $x$ & $x$ & $x$ & $x$ & $x$ & $x$ & टा? \\
\hline $\mathrm{HX}+\mathrm{TP}-7$ & & $x$ & $x$ & $x$ & & & & & & (2) \\
\hline $\mathrm{HX}+\mathrm{TP}-8$ & & $x$ & $x$ & $\dot{x}$ & & & & & & d \\
\hline
\end{tabular}

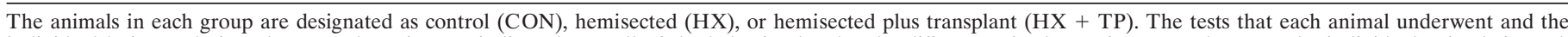

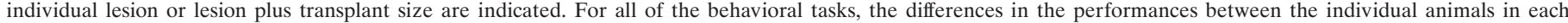
particular group could not be attributed to differences in either lesion size or the extent of the transplant apposition.

flexion of their abdominal muscles resulting in excessive elevation of the pelvis from the surface. Emerging abilities to roll over were impeded by limited right forelimb movement. By P10-11 (mean day of maturation of body righting for normal rats), significant impairments in motor control were evident in lesion-only rats as compared with the $\mathrm{CON}$ group (Fig. $2 B$; HX rating scale $=2.3 \pm$ 0.52 vs $\mathrm{CON}$ rating scale $=5.0 ; p<0.05)$. By the third week of life, isolated rotation was still minimal (Fig. $2 A ; \mathrm{P} 14.8 \pm 2.9$, significantly different from CON; $p<0.05$ ). This abnormal motor pattern was used consistently by all lesion-only rats and was evident in those with the smallest and largest transverse extent of lesion. Although the response was aberrant, it was considered mature, because there was little subsequent improvement in motor performance for body righting.

The presence of a transplant at the cervical lesion site accelerated the time of development of the righting reflex toward normal and permitted the establishment of normal movement patterns. Transplant animals initially used random motor patterns in righting. The righting response matured during the second week, at which time all transplant rats incorporated upper body rotation, forelimb external rotation, paw placement on the surface, and pelvic rotation to turn over. This sequence of righting movements in $\mathrm{HX}+\mathrm{TP}$ animals resembled normal motor patterns (Fig. $2 A$; CON P10.6 \pm 1.2 vs $\mathrm{HX}+\mathrm{TP} \mathrm{P} 13 \pm 2.9 ; p>$ $0.05)$, although the maturation of righting was delayed slightly. The quality of the body movements used by transplant rats to right was reduced significantly compared with normal rats (Fig. $2 B ; \mathrm{CON}$ rating scale $=5$ vs $\mathrm{HX}+\mathrm{TP}$ rating scale $=4 ; p<0.05)$, but was significantly better when compared with lesion alone (HX rating scale $=2.3 \pm 0.52$ vs $\mathrm{HX}+\mathrm{TP}$ rating scale $=4 ; p<0.05)$.

\section{Spatial orientation}

Normal rats repositioned themselves to face upward when they were inverted on a board inclined to a $30^{\circ}$ angle. Initial placement on the board elicited immediate attempts to achieve postural stability. Once secure in their stance, rats easily transferred body weight from one limb to another to coordinate reciprocal smooth repositioning of all limbs to turn $90^{\circ}$ (i.e., placing their body perpendicular to the angle of incline). Once facing upward, they ascended to the top edge of the board using an alternating, rhythmical locomotor pattern. Normal development of this response occurred by P11 (Fig. 3; CON rating scale $=5$ ).

Lesion-only rats were unable to support their body weight on all four extremities when they were placed on the angled board. 
A
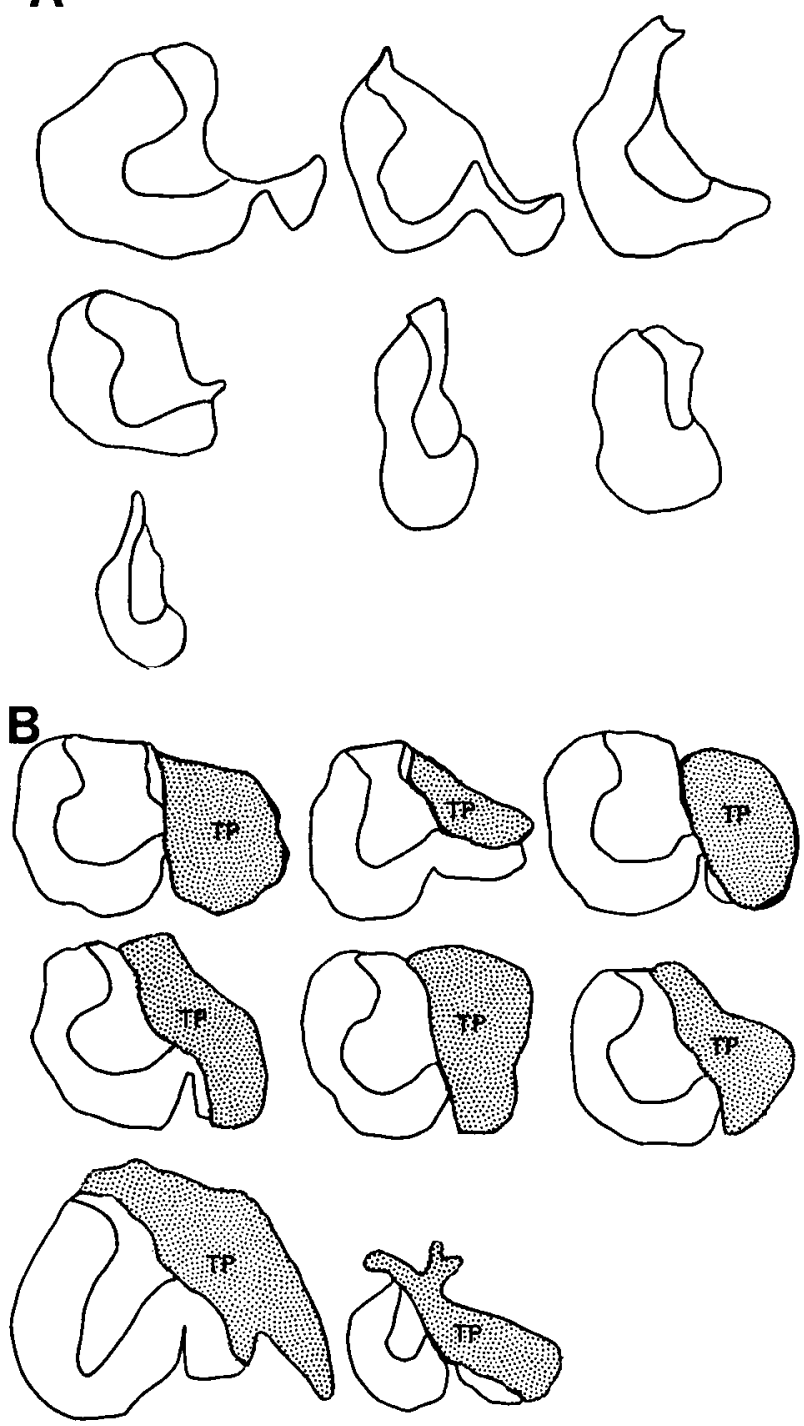

Figure 1. Lesion or lesion plus transplant sites in rats used for behavioral analysis. Host tissue remaining in each lesion-only $(A)$ or lesion plus transplant rat $(B)$ through the site of greatest injury extent. The reconstructed lesion sites show a similar transverse extent of the injury in lesion-only and lesion plus transplant animals (see Materials and Methods for lesion criteria). In each transplant rat $(B)$, the transplants $(T P)$ are well apposed to the host cord.

Instead, during the first 14 postnatal days, they firmly pushed their nose into the board and widened their hindlimb base of support to maintain a static posture. Transferring of body weight was impaired in this group; consequently, any attempt to reorient the body by repositioning the limbs was unsuccessful, and the rats rolled off of the board. From the third to fourth postnatal weeks, all HX rats substituted novel motor patterns for the normal motor sequence used to turn upright. For example, five of six HX rats laterally flexed their trunk and swiftly pivoted their hindlimbs around their stationary upper body, making final postural adjustments with the left forelimb. This motor pattern was the reverse of that used by normal rats (Fig. 3; CON rating scale $=5$ vs $\mathrm{HX}$ rating scale $=2.5 \pm 1.0 ; p<0.05)$. Use of right forelimb to assist in turning or in final body placement was minimal or absent in all $\mathrm{HX}$ rats.
Initially in the lesion plus transplant animals, attempts to reposition any limb on the board caused these rats to roll off of the board. By the end of the first postnatal week, however, they were able to turn on the inclined board by sequencing left forelimb lateral stepping with subsequent hindlimb movements, which suggests the development of abilities to shift body weight to maintain balance. During the second week, seven of eight rats realigned their body initially through minor positional changes (e.g., lateral stepping) followed by moving the right forelimb forward, laterally flexing the trunk, and stepping with both hindlimbs. Forelimb and upper body movements preceded hindlimb and lower body movements, similar to the normal pattern (Fig. 3; $\mathrm{CON}$ rating scale $=5 \mathrm{vs} \mathrm{HX}+\mathrm{TP}$ rating scale $=4.1 \pm 0.64 ; p>$ $0.05)$. In response to loss of balance, transplant rats reacted automatically to stabilize themselves by widening their hindlimb base of support and abruptly extending all limbs. In contrast, lesion-only animals lost control and rolled off of the board. This is one example of movement patterns used by the HX + TP group that were significantly better than those used by the HX group (Fig. 3; $\mathrm{HX}+\mathrm{TP}$ rating scale $=4.1 \pm 0.64 \mathrm{vs} \mathrm{HX}$ rating scale $=$ $2.5 \pm 1.0 ; p<0.05)$.

\section{Development of postural reflexes and forelimb movements: motor skills that are prerequisite for target-directed reaching}

\section{Chin placing}

Chin-placing responses are absent at birth in normal rats. During the first postnatal week in the normal rat, only small, random, bilateral forelimb movements were elicited in response to stimulation of the ventral surface of the chin. Chin placing developed during the second postnatal week when, in response to stimulation of the ventral surface of the chin, one or both forelimbs were placed reciprocally or simultaneously onto the surface (Fig. 4A, CON).

The typical time course for the development of chin placing in the three groups is illustrated in Figure $4 \mathrm{~B}$. Lesion-only rats responded to stimulation on their chin with an exaggerated version of the normal immature response (Fig. $4 B$; HX, forelimb use $=1.0$; open squares at $\mathrm{P} 6-11 ; p<0.05)$. For example, the rats struggled, rotated their heads, and infrequently and inconsistently moved their left forelimbs randomly. By P11, although there was a temporal delay between application of the stimulus and the motor response, the frequency of the random left forelimb movements increased and occasionally the limb was placed on the surface. By the third postnatal week, all HX rats immediately placed their left forelimbs onto the surface in response to the stimulus, indicating partial maturation of the reflex (Fig. $4 A$; $\mathrm{HX}$ P14.5 $\pm 1.0 ; p<0.05$; and Fig. $4 B$ ). Lesion-only rats never developed a bilateral forelimb response to stimulation under the chin and therefore never achieved the mature response.

Transplantation after neonatal spinal cord injury prevented the delay in the development of chin placing. In the first week of life, the reaction of $\mathrm{HX}+\mathrm{TP}$ rats was similar to that of normals; they responded to the stimulus with head rotation, lateral trunk flexion to either side, and random movements of both forelimbs (Fig. 4B, open triangles; P6-8). By P13, the response matured (Fig. 4A; $13.2 \pm 3.4)$, and one or both forelimbs were placed on the surface in addition to the chin (Fig. $4 B$; $\mathrm{HX}+\mathrm{TP}$; forelimb use $=1.5 \pm$ 0.53). Placing in the $\mathrm{HX}+\mathrm{TP}$ group was characterized by a momentary delay between application of the stimulus and limb placement, distinguishing the response from that in normal animals. Unlike HX animals, however, all of the HX + TP rats were 
A

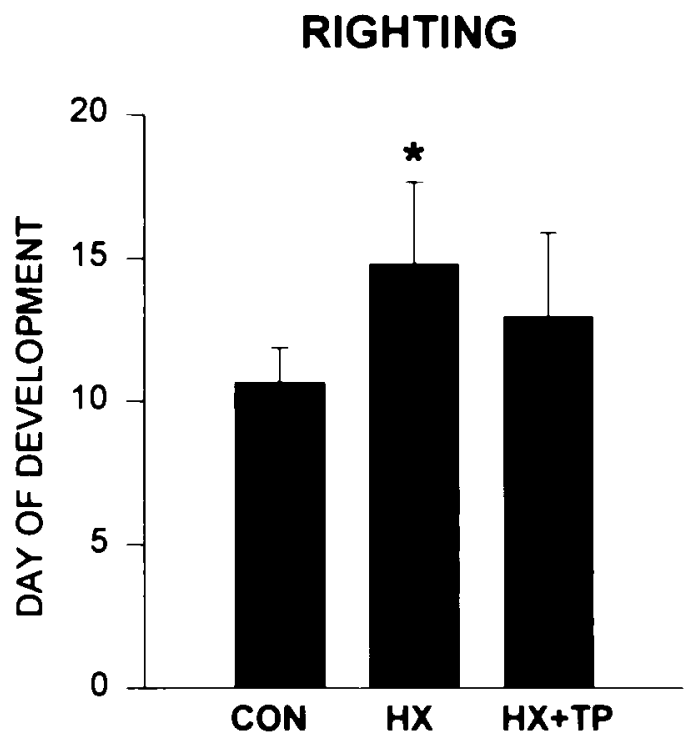

B

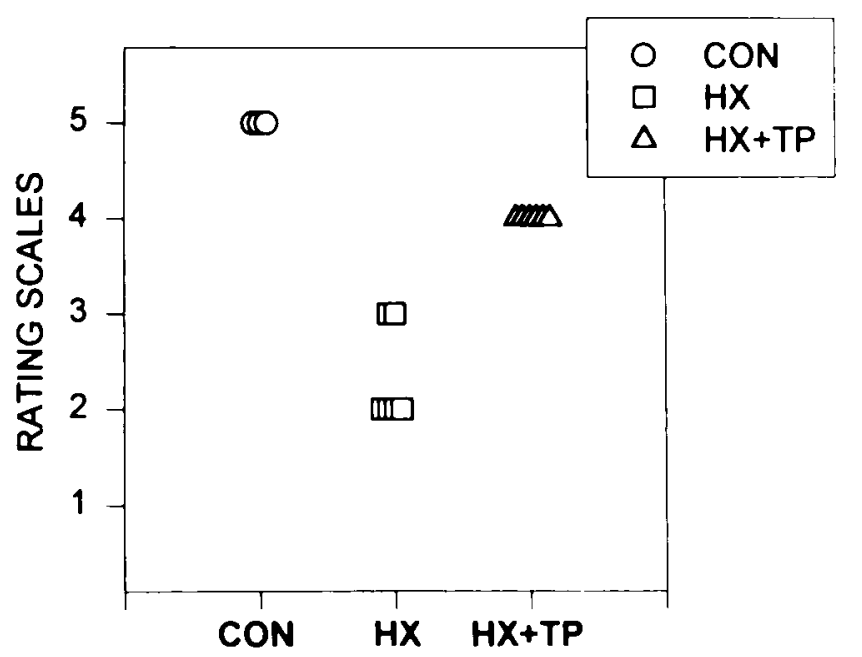

Figure 2. Effect of neonatal cervical spinal cord injury and transplantation on postnatal development of the righting reflex. $A$, Histogram comparing average $( \pm \mathrm{SD})$ postnatal day at which the mature reflex develops in normal uninjured (CON, $n=9)$, hemisected $(H X, n=6)$, and hemisected plus transplant $(H X+T P, n=8)$ rats. The righting reflex matures significantly later in $\mathrm{HX}$ rats as compared with CON rats $(p<0.05)$. HX + TP rats demonstrate a significant improvement compared with HX rats $(p<0.05)$, because righting develops in a time course similar to that of CON rats. $B$, Scatter plot of upper body/forelimb use for righting at P11. Each symbol represents an individual animal in the specified group. Righting matures in CON rats by the P11, whereas HX and HX + TP rats still exhibit immature behaviors. To initiate turning over, $\mathrm{CON}$ rats use upper body rotation in advance of lower body rotation (rating scale $=5$ ). In their attempt to rotate the body, HX rats instead excessively flex their pelvis and engage in other strategies to roll over (rating scales $=2$ and 3 ), representing a different strategy compared with $\mathrm{CON}$ and $\mathrm{HX}+\mathrm{TP}$ rats. $\mathrm{HX}+\mathrm{TP}$ rats demonstrate emerging abilities to rotate their bodies smoothly from supine to prone but require multiple rotations before achieving the prone position (rating scale $=4$ ). This represents a marked alteration in upper body/ forelimb skill development for the righting reflex compared with CON rats.
SPATIAL ORIENTATION

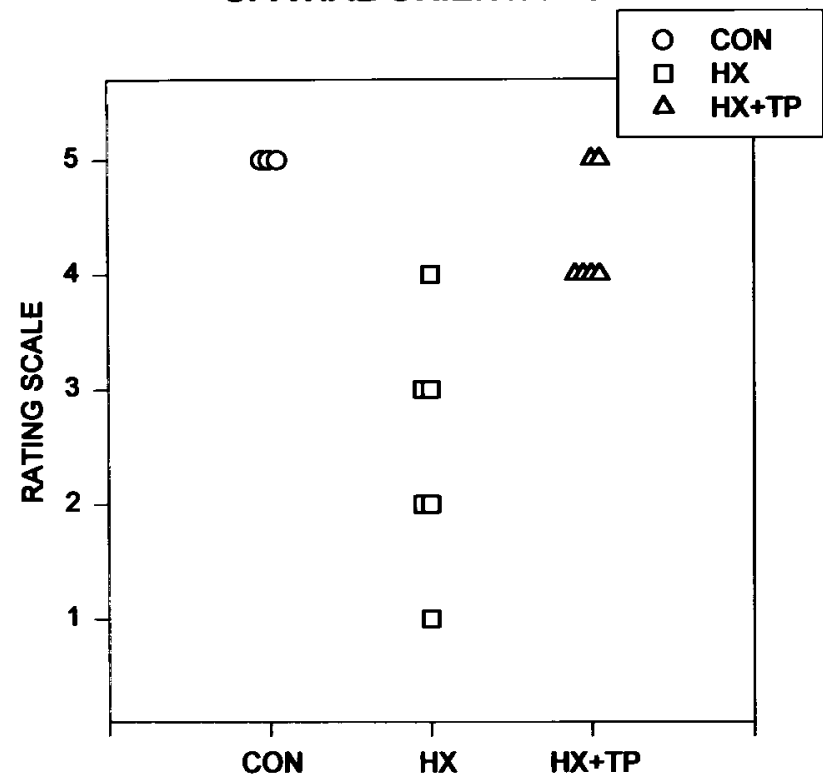

Figure 3. Effect of neonatal cervical spinal cord injury on the ability to orient the body upright during the second postnatal week. Each symbol represents an individual animal in the specified group. As normal uninjured rats mature, they turn their bodies on an inclined board to orient their head upward by first moving their forelimbs and then realigning their body with their hindlimbs. $H X$ rats develop alternative strategies for failed development of isolated forelimb movements and respond with movement substitutions to accomplish the goal. These aberrant movements are different from the pattern used by both $C O N$ and $H X+T P$ rats. $\mathrm{HX}+\mathrm{TP}$ rats, although delayed, develop the ability to turn themselves around on an inclined board, using a developmental sequence similar to that of normal rats rather than the substitutions used by HX rats.

able to respond with both forelimbs ( $\mathrm{HX}$ rating scale $=1.0 \mathrm{vs} \mathrm{HX}$ $+\mathrm{TP}$ rating scale $=1.5 ; p>0.05)$.

\section{Grasping and proximal control}

A mild obligatory grasp reflex that was elicited in all rats at birth became integrated in normal rats during the first postnatal week when active grasping developed (P6). The strength of active grasp was measured functionally by evaluating rats during strenuous activities that incorporated grasping with proximal control. Proximal control, also a prerequisite for target-directed reaching, was achieved through active use of the muscles surrounding the shoulder joint and associated stabilizing movements of the upper and lower body. The development of proximal stability was measured by observing the rats supporting their body weight (chin-up) when grasping a horizontal rod. Normal rats exhibited proximal control of the upper body by the beginning of the second week of life (Fig. $5 A, C O N)$. During the second week of life, muscle strength, proximal stability, and control over the body improved, enabling the rat not only to sustain a static chin-up but also to reposition the forelimbs along the rod (Fig. $5 A$, open circles; rating scale $=$ 5.0). Over the next 2 weeks, normal animals narrowed their base of support on the rod, sustained their grip for increasing periods of time, and transferred to the opposite side of the rod.

Neonatal cervical spinal cord overhemisection disrupted the development of volitional grasping, proximal control, and isolated use of muscles around the shoulder (Fig. $5 A, H X$ ). During the second and third postnatal weeks, three of five HX rats used an obligatory grasp reflex to wrap their digits around the rod (Fig. $A$; 
A

\section{CHIN PLACING REFLEX}

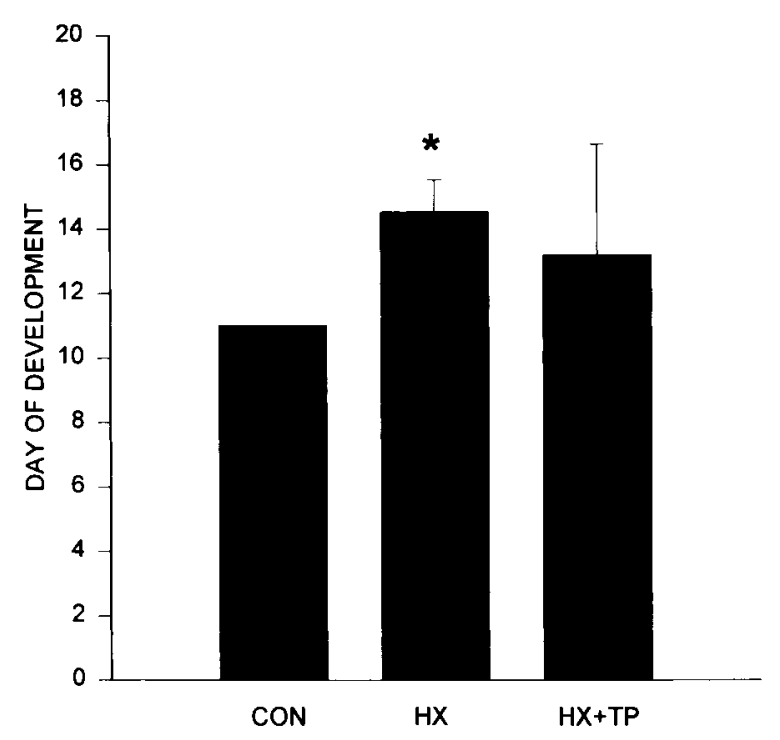

B

CHIN PLACING

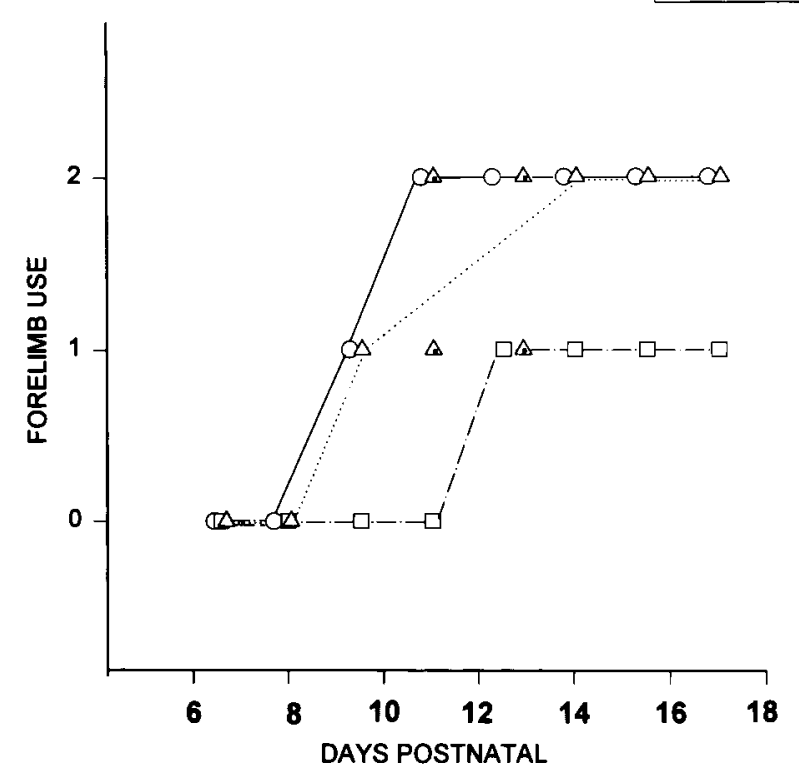

Figure 4. Effect of neonatal cervical spinal cord injury on the development of chin placing. $A$, Comparison of the day of maturation of the reflex $(x$-axis $=D A Y S$ POSTNATAL $)$. Both $H X$ and $H X+T P$ rats develop the paw-placing response to stimuli to the chin later than $C O N$ rats. The delay in maturation of the response is more pronounced in HX rats $(p<0.05)$ than in $\mathrm{HX}+\mathrm{TP}$ rats. $B$, Comparison of the development of the chin-placing reflex in normal $(C O N)$ and lesion with $(H X+T P)$ or without $(H X)$ transplantation. Each open symbol represents the response of all animals in the specified group. Symbols with the center marked represent $50 \%$ of the animals. CON rats place both forelimbs on the surface during the second week of life in response to a tactile stimulus to the chin (forelimb use =2). The onset of the response is delayed in HX rats, and the mature response never develops. Instead, the mature response of $\mathrm{HX}$ rats is to place the left forelimb on the surface, with occasional random rating scale $=1.0$ ), they but lacked the proximal control to pull to the chin-up posture. Instead, their attempts to contract their muscles resulted in movement substitutions including excessive abdominal flexion with the hindlimbs swinging up to grip the rod, which consequently inverted the rats (Fig. 5A, open squares; rating scale $=1.0)$. At this 2 week period, the two other rats in the group demonstrated emerging capabilities to execute the motor sequences that are necessary to accomplish a chin-up position (2 weeks) (Fig. $5 A$, open squares; rating scale $=3.0$ and 4.0 ). In contrast to normal developing rats, the base of support used by HX rats was wide (approximately the width of their shoulders), and the forelimbs were not repositioned on the rod, indicating weakness in flexor musculature and a predominant grasp reflex (Fig. 5A; significantly different from $\mathrm{CON}, p<0.05$ ). By maturity (P31), HX rats typically either used a moderately wide base of support on the rod or released one forelimb, sustaining their grasp with the other and alternating between hanging and momentarily pulling to a chin-up.

$\mathrm{HX}+\mathrm{TP}$ rats developed the ability to achieve and maintain the chin-up posture, although the emergence of mature skills was delayed and varied within the group. Differences in motor performance in the HX + TP animals could not be attributed to differences in the transverse extent of the lesion. All HX + TP rats grasped and hung from the pole until proximal control improved and they could pull to a chin-up (Fig. $5 A, 2$ weeks postnatal, open triangles; $n=4$ at rating scale $=4.0$; significantly different from CON, $p<0.05)$. At 2 weeks postnatal, two $\mathrm{HX}+$ $\mathrm{TP}$ rats were more precocious than others in the group: they developed full proximal control to achieve a chin-up (Fig. $5 A$, open triangles; at rating scale $=5.0)$. Although the performance of all $\mathrm{HX}+\mathrm{TP}$ rats resembled normals by the fourth week of life, there were differences in the strategy for accomplishing the task. For example, although the left forelimb maintained its original grasp, the base of support on the rod widened because the right forelimb slid laterally. As proximal control developed over the third postnatal week, the rat could return the right forelimb to a neutral position or could adjust total body position to evenly redistribute (recenter) body weight between the forelimbs. Similar to normal rats, by P31 HX + TP rats narrowed their base of support and repositioned both forepaws laterally along the rod.

\section{Grooming}

A noxious (cold) stimulus was applied to the bridge of each rat's nose to evaluate active range of motion and postural stability requisite for functional skills such as grooming. In normal rats, forelimb responses to remove the stimulus were not developed at birth. Instead, the initial responses included a combination of retraction of the head and body or pivotal movements with the forelimbs to escape from the stimulus. These motor patterns were categorized as immature (Fig. 5B, open symbols). The use of one forelimb was observed occasionally and was recorded as partially mature (Fig. 5B, partially opened circle at P4). By P6, the response matured and a bilateral swiping motion was used to displace the stimulus (Fig. 5B, filled circles from P6 to adulthood). The quality

$\leftarrow$

unproductive right forelimb movements (forelimb use $=1$ ), which is a markedly different strategy from that used by CON and HX + TP rats. $\mathrm{HX}+\mathrm{TP}$ rats follow a similar sequence of development as compared with CON, but do so with a slight developmental delay. By 2 weeks postnatal, however, all transplant rats place both forepaws on the surface in response to the stimulus. 
A

\section{PROXIMAL CONTROL WHILE GRIPPING A CYLINDRICAL POLE}

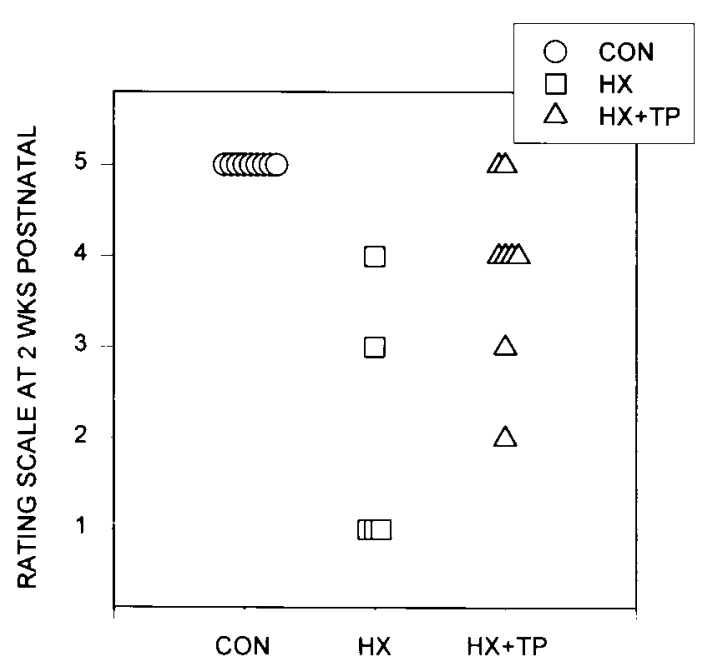

B

\section{FORELIMB MOVEMENTS TOWARD MIOLINE IN RESPONSE TO A COLD STIMULUS}

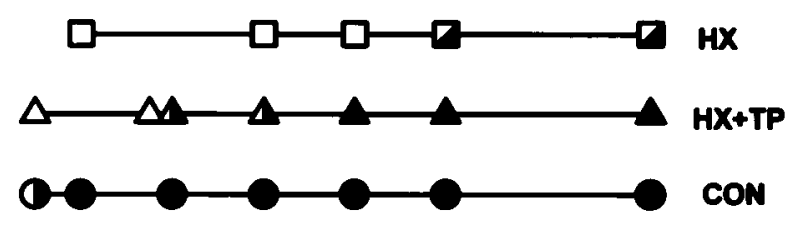

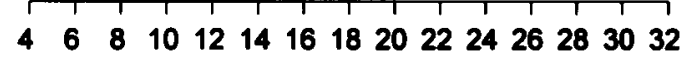
DAYS POSTNATAL

Figure 5. Effect of neonatal cervical spinal cord injury on the development of proximal control during static grasping and dynamic forelimb use. $A$, Proximal control for achieving and maintaining a chin-up at 2 weeks postnatal. Rating scores range from 1 to $5: 1$ indicates the most immature and aberrant response, 5 represents maturation of the response, and the intermediate scores represent progressive development of skills for this task. Each symbol represents an individual animal in the specified group. CON rats use proximal muscle control to achieve and maintain a chin-up. HX rats never maintain a chin-up and instead achieve various intermediate levels of control, often using their obligatory right grasp reflex to sustain their grip. Mostly, when HX rats attempt to support their body weight, they excessively flex their pelvis, swinging their hindlimbs up onto the pole. Even with this excessive effort, they do not maintain their grasp and soon drop to the surface (rating scale $=1$ ). Although the $\mathrm{HX}+\mathrm{TP}$ rats initially drop to the surface, unlike the HX rats they eventually develop the motor components for the mature response. Most HX + TP rats use both forelimbs to hold onto the pole and use proximal control to achieve a chin-up (rating scale $=4$ ), although others use varying degrees of normal behavior. $B$, Forelimb use to groom the face. Each symbol represents the typical response of the animals in the specified group. Filled symbols indicate mature behavior. Open symbols indicate immature behavior. Partially filled symbols indicate emerging mature behaviors. Unlike CON rats $(n=9)$, HX rats $(n=6)$ never develop mature use of the and accuracy of the swiping motion improved during the second and third week of life and developed into a grooming pattern (i.e., forelimbs move through quadrants I-II or III-IV; see Materials and Methods).

Lesion-only rats failed to develop mature bilateral forelimb movements in response to the stimulus and instead substituted immature avoidance patterns and compensatory strategies. Forelimb swiping movements were not used; instead, immature behaviors such as head retraction, pivoting, or walking away from the noxious stimulus persisted (Fig. 5B, open squares). By P22, $\mathrm{HX}$ rats used only the left forelimb to swipe at the stimulus (Fig. $5 B$; partial mature response). Right forelimb adduction failed to develop. HX rats never developed bilateral forelimb use in midline (Fig. 5B, no completely filled squares).

The mature bilateral response developed in transplant rats (Fig. 5B, filled triangles), although the onset and maturation of the response was delayed compared with normals (CON maturation at P6 vs $\mathrm{HX}+\mathrm{TP}$ maturation at P18). The immature response pattern was present during the first week (Fig. 5B, open triangles). A partial mature response developed during the second postnatal week (Fig. 5B, partially filled triangle representing left forelimb used for batting and swiping at the stimulus). By P18, all transplant rats consistently used both forelimbs (reciprocally or symmetrically) to groom their faces. Most transplant rats lacked full supination and therefore could only rub their faces with the medial side of their paws. The frequency and quality of movement improved during the fourth postnatal week. In HX + TP rats, right and left forelimb movements into quadrants I and II (see Materials and Methods) was 72 and 98\%, respectively, of normal forelimb movement. This represents a dramatic improvement when compared with the lesion-only animals, who do not use the right forelimb at all and use the left forelimb at only $37 \%$ of the frequency of normal rats.

\section{Stepping over obstacles}

Qualitative assessments indicated that overground locomotion developed in all rats by the end of the second week of life. Normal rats walked earlier and with better form than the lesion or lesion plus transplant animals (Bregman and Goldberger, 1983; KunkelBagden and Bregman, 1990; Kunkel-Bagden et al., 1992; Diener, unpublished data). During the third and fourth week of life, the ability of each rat to anticipate and step over obstacles placed in its path was evaluated. The ability to step over the obstacle requires anticipatory postural adjustments, proximal and distal muscle control, and accurate aiming of the lifting limb. These forelimb skills and postural reactions are also prerequisites for smooth, coordinated reaching. Normal animals walked easily overground, anticipating and clearing each obstacle with all four extremities (Fig. 6; $C O N$ rating scale $=3.0$ ). Lesion-only rats walked overground but demonstrated significant deficits as compared with $\mathrm{CON}$ in anticipating and stepping over obstacles (Fig. 6; $H X$ rating scale $=1.0 ; p<0.05)$. For example, $\mathrm{HX}$ rats did not lift their right forelimb voluntarily over the obstacle. Instead, the forward movement of the body caused the right forelimb to

forelimbs for grooming. Immature responses (e.g., backing away from a noxious stimulus) are used initially, and later emerging mature behaviors (e.g., only the left forelimb bats at the stimulus) develop in HX rats. Although HX + TP rats $(n=8)$ demonstrate a prolonged period during which they exhibit immature behaviors, they all develop the motor skills required for maturation of the grooming response. 


\section{MANEUVERING OVER OBSTACLES}

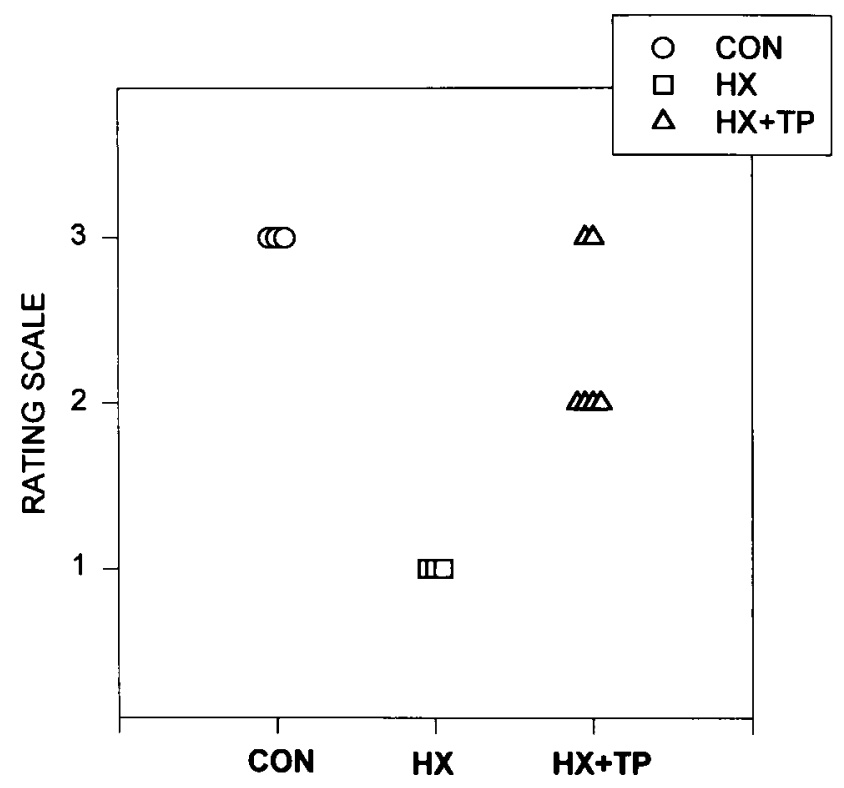

Figure 6. Effect of neonatal cervical spinal cord injury on the acquisition of precise right forelimb placement during locomotion. $C O N(n=3) ; H X$ $(n=4) ; H X+T P(n=6)$. Rating scale ranges from 1 to 3: 3 represents mature behavior and 1-2 are degrees of aberrant movements. Each symbol represents an individual animal in the specified group. CON rats step over obstacles in their path during overground locomotion. CON rats anticipate the obstacle and raise their forepaws to completely clear the height of the obstacle during swing-through. HX rats demonstrate significant deficits compared with both $\mathrm{CON}$ and $\mathrm{HX}+\mathrm{TP}$ rats: they fail to anticipate the obstacles and instead drag their forelimbs and place them over the obstacle. In contrast, most HX + TP rats anticipate the obstacle but fail to sufficiently clear it during the swing phase, contacting the distal tip of their paw to the upper edge of the obstacle.

contact the obstacle. After contact, the limb was placed reflexively over the obstacle in a pattern resembling forelimb proprioceptive placing. This movement also resembled a dragging motion that ended with either the ventral or dorsal surface of the paw on the runway. Both hindlimbs and the left forelimb of HX rats usually anticipated and cleared the obstacle. Initially, HX + $\mathrm{TP}$ rats dragged the right forelimb over the obstacle but soon developed the ability to anticipate and step over it. This response was a significant improvement compared with the response of the lesion-only animals (Fig. 6; $H X$ rating scale $=1.0$ vs $H X+T P$ rating scale $=2.3 \pm 0.5 ; p<0.05)$. Secondary to reduced forelimb range for shoulder flexion during the swing phase, most (four of six) of transplant rats brushed the tip of their right forepaws on the top edge of the obstacle as the forelimb stepped over. The inability to lift the right forelimb completely over the barrier distinguished transplant rats from normals, although the difference was not significant.

Forelimb use in adult rats: target-directed reaching and associated postural adjustments in adult animals Sticker removal

Forelimb movements used to remove a sticker from the head were similar to grooming motions. Normal rats swiftly reached for, grasped, and abruptly pulled the sticker from the head using either or both forelimbs. Balance was maintained by shifting body weight backward toward the midtrunk (Figs. 7, 8).

Lesion-only rats exhibited significant deficits in sticker removal bilaterally (Figs. $7 B, C, 8, C O N T R A L A T E R A L$ rating scale $=$ $3.8 \pm 0.45$ and IPSIL ATERAL rating scale $=1.2 \pm 0.84 ; p<$ $0.05)$. Only the left forelimb was raised to the height of the sticker (Fig. 7B,C). Grasping failed to develop; the radial and dorsal surface of the left forepaw rubbed at the sticker, loosening but never removing it (Figs. $7 B, C, 8, C O N T R A L A T E R A L$ rating scale $=3.8$ ). Deficits in balance were evident during this task. When they raised both forelimbs to the sticker, HX rats often fell to one side. To compensate for the compromised balance reactions, postural control was acquired through the use of a threepoint stance (not pictured) or by altering the hindlimb base of support and counterbalancing with the right extremity moving across the midline while the left forelimb was raised to the sticker (Fig. 7B,C).

Transplant rats removed the sticker but lacked the speed, smooth coordination, and powerful grasp of normals (Fig. 8, $H X+T P, C O N T R A L A T E R A L$ rating scale $=5.0$ and IPSILAT$E R A L$ rating scale $=4 \pm 1.1 ; p<0.05)$, yet they were significantly better than HX animals $(p<0.05)$. In contrast to normal rats, $\mathrm{HX}+\mathrm{TP}$ rats required multiple reaching attempts to remove the sticker. Because grasping was impaired bilaterally, much of the sticker was dislodged initially from the face by repetitive rubbing before it was grasped and removed (Fig. 7D, $E)$. Although the movement pattern was similar, HX + TP rats used their left forelimb more than their right (compare scores on Fig. 8, CONTRALATERAL rating scale $=5.0$ and IPSILAT$E R A L$ rating scale $=4 \pm 1.1$ ). Similar to $\mathrm{CON}$ rats, $\mathrm{HX}+\mathrm{TP}$ rats shifted their body weight backward to maintain balance during reaching [compare rats in Fig. $7 A(C O N), D, E(H X+$ $T P)]$. These results indicate that after cervical spinal cord lesions, transplants not only mediate recovery of rhythmic alternating movements such as those in locomotion (Kunkel-Bagden and Bregman, 1990; Bregman et al., 1993), but they also mediate recovery of skilled forelimb movements.

\section{Target reaching}

Normal rats used appropriate postural reactions when using either forelimb to reach for a target at a distance from the body. To lift one forelimb from its resting posture in stance, the rat transferred its weight to the opposite forelimb as well as the lower trunk/pelvis. They then actively extended their nonweighted forelimb through a vertical grate to reach a series of horizontal shelves (wells) containing $45 \mathrm{mg}$ food pellets. The reaching limb adducted and externally rotated as it raised (Fig. 9A) and then extended (Fig. 9B) into the well. The forelimb pronated and the digits extended over the pellet, anticipating pellet retrieval (Fig. $9 B)$. Next, the digits of the forelimb grasped the pellet, supinated slightly, and withdrew from the shelf (Fig. 9C) The forelimb further supinated as it approached the mouth, and the contralateral forelimb mirrored its position to assist in holding the pellet under the mouth to eat (Fig. 9D). Normal animals reached more accurately toward a target and consumed more pellets than either experimental group (see Fig. $11 A, B$ ) and never used compensatory strategies (Fig. 10, CON). Normal animals did not exhibit a dominant limb for reaching (Fig. 11).

Skilled reaching and associated postural adjustments failed to develop in lesion-only rats. Occasionally these rats fell to one side either while obtaining food or returning to stance after food retrieval. Food was obtained by substituting tongue extension for absent reaching skills (Figs. 9E,F, 10, bar at 0\%). Furthermore, once the pellet was on the tongue and ready to be eaten, impaired bilateral forelimb use was pronounced; the right paw failed to 

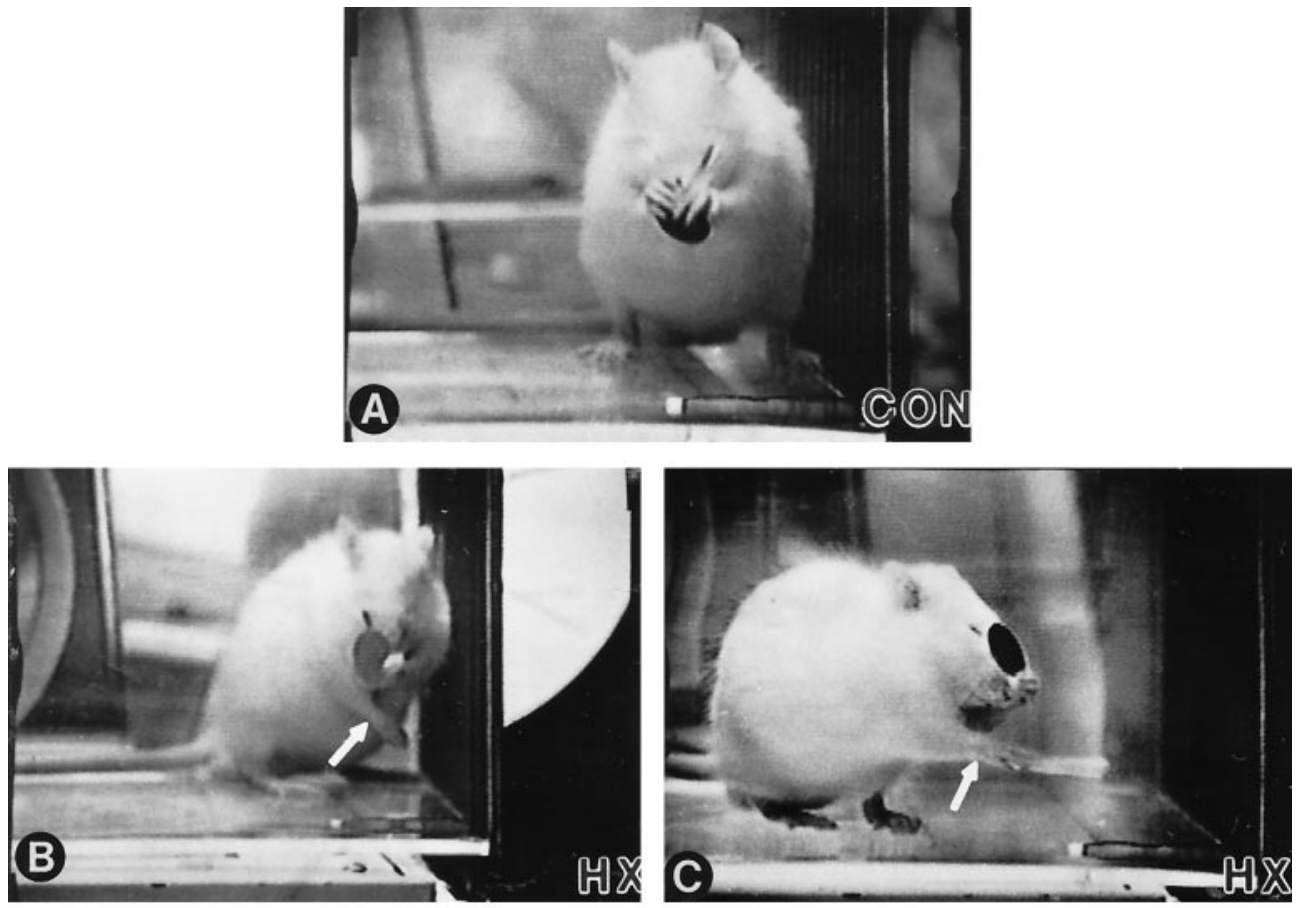

Figure 7. Comparison of representative responses for sticker removal in each group of rats. $A, C O N$ rats use a bilateral approach to remove the sticker, with both forepaws gripping and pulling the sticker from the nose. Simultaneously, they maintain a stable base with both hindlimbs slightly externally rotated. $B$, $C$, Two different rats representing the typical response of the $H X$ group. Each uses aberrant strategies when attempting to dislodge the sticker. To maintain balance, the right forelimb (arrows) moves
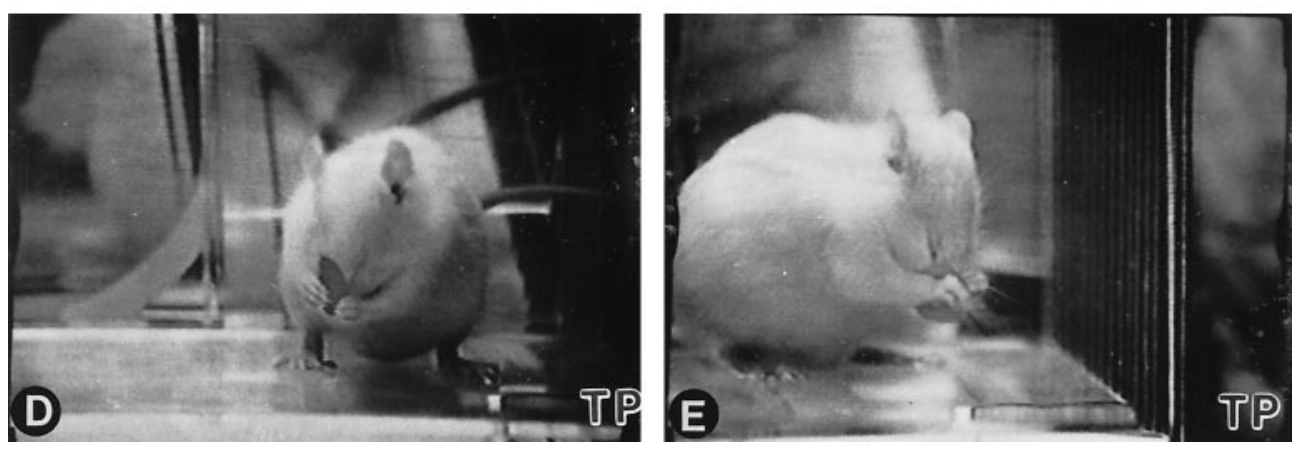
forward and across midline while the left forelimb approaches the sticker. The photographs also depict the usual posture of the rats in the HX group (i.e., slightly contorted body position with the hindlimbs asymmetrically placed and the upper body frequently rotated in opposition to the lower body). $D, E$, Two representative $H X+T P$ rats demonstrating the response consistently observed in this group. Similar to CON rats, the HX + TP rats use both forelimbs to reach for the sticker while maintaining a stable base of support.

approach midline to assist in holding the pellet (Fig. 9F). This movement pattern frequently resulted in awkward posturing, including body contortions and frequent loss of balance (Fig. 12). The use of compensatory strategies limited the range over which HX rats could accumulate pellets; consequently, significantly fewer pellets were taken and consumed as compared with normal and transplant rats (Fig. 11A,B; $p<0.05$ ).

Although qualitatively different from normal rats, target reaching developed in $\mathrm{HX}+\mathrm{TP}$ rats (Fig. 10; score $=84 \%$ ). Despite bilateral forelimb deficits, $\mathrm{HX}+\mathrm{TP}$ rats preferred to use their left limbs for reaching. Only when the pellets rested on the very outer edge of a shelf did the CON and HX + TP rats use their tongues for pellet retrieval (Fig. 11). Typically, one forelimb (left more than right) entered the shelf (Fig. $9 G$ ) to retrieve the pellet (Fig. 9H) while the opposite forelimb remained on the ground (Fig. 9G) or on the edge of a shelf for support (Fig. 9H). Forelimb pronation was occasionally excessive compared with that of normal animals and was accompanied by extreme digit extension over the pellet. Aiming of the forelimb was not always smooth or directed toward any particular pellet or shelf; often the left forelimb was inserted and withdrawn repetitively before a successful grasp was achieved (significant improvement over HX animals; $p<0.05$ ). Occasionally after the rat grasped with the right forepaw, the pellet dropped from the paw as the limb was withdrawn from the shelf. After successful withdrawal of the grasped pellet, both forelimbs came to midline under the mouth to hold the pellet to eat (Fig. 9I). The HX + TP rats usually shifted their weight backward and relied on other postural adjustments associated with reaching in a manner similar to that of normal animals (Fig. 12). For example, HX + TP rats used the nonreaching forelimb on a shelf to maintain balance while the opposite forelimb reached for and grasped a pellet. HX + TP rats resembled normals in their approach to reach for, grasp, withdraw, and ingest the pellet (compare Fig. 9, $A-D$ with $G-I$ ). Despite some qualitative differences in reaching styles, $\mathrm{HX}+\mathrm{TP}$ rats used their forelimbs to grasp significantly more pellets than HX rats in a time frame more consistent with but not identical to that of normals (Fig. 11A,B). Thus, transplants supported the development and maturation of skilled forelimb function in goaldirected reaching to targets in space as well as on the body. The qualitative differences in execution of the reaching movements in $\mathrm{HX}+\mathrm{TP}$ animals as compared with control animals suggest that there may be differences in the descending supraspinal input controlling reaching movements (Diener and Bregman, 1998).

\section{Summary}

Neonatal cervical spinal cord overhemisection retarded the onset of forelimb motor control and resulted in the production of 


\section{STICKER REMOVAL}

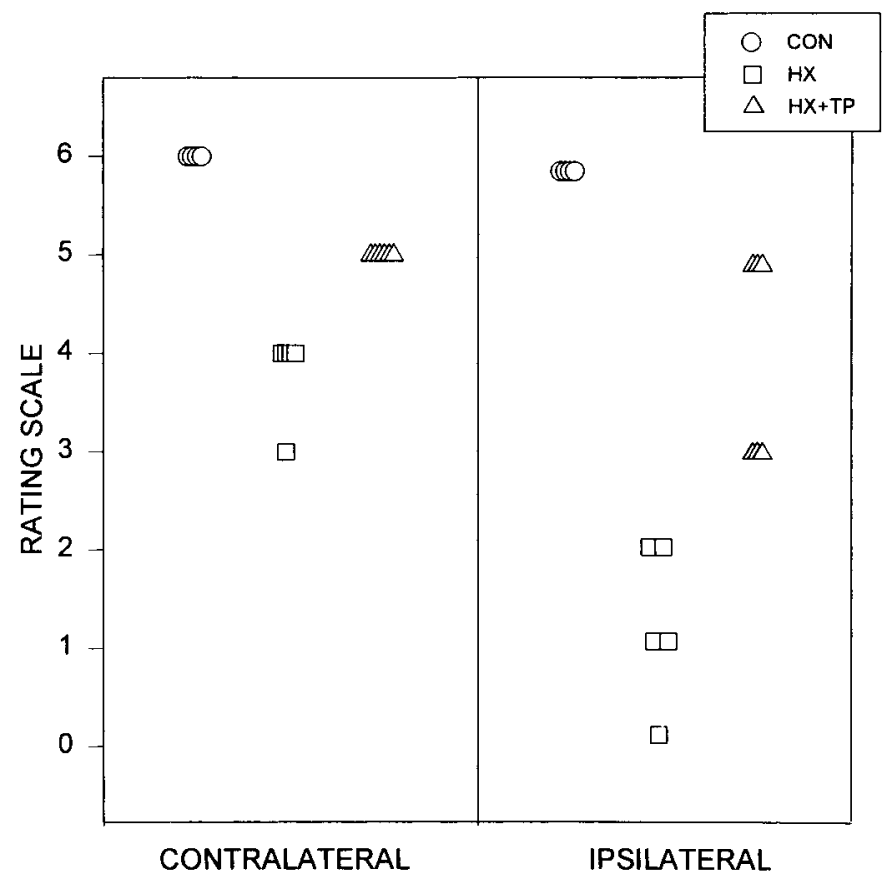

Figure 8. Effect of neonatal cervical spinal cord injury on reaching for targets on the body. Rating scales range from 0 to 6: 6 represents mature behavior (i.e., full range of motion), 0 represents abnormal behavior (i.e., little to no movement), and intermediate numbers reflect degrees of active range of motion of each forelimb, analyzed individually. Each symbol represents the specified forelimb of an individual animal. CON rats quickly and consistently use either or both forelimbs to remove a sticker placed between the eyes. $H X$ rats $(n=5)$ demonstrate marked deficits in target reaching compared with $\mathrm{CON}$ rats and $H X+T P$ rats. Their best response is to bring their left (contralateral) forelimb to touch the sticker. The right (ipsilateral) forelimb, at best, moves up to the level of the sticker, but the limb remains lateral and fails to contact the sticker. The HX + TP rats $(n=6)$ use either or both forelimbs to remove the sticker. Because they require multiple attempts to free the stickers from their faces, the HX + TP rats exhibit obvious deficits in forelimb motor control as related to speed and dexterity when compared with $\mathrm{CON}$ animals.

abnormal motor patterns. Lesion-only rats also failed to develop forelimb goal-directed movements or associated postural adjustments and instead used compensatory behaviors. In contrast, after neonatal spinal cord lesion plus transplants, there was a short temporal delay in the development of some postural reflexes compared with normal rats, but the forelimb motor patterns developed and resembled those used by normal rats. Transplant rats developed both forelimb use for target-directed reaching and appropriate associated postural adjustments to sustain balance during movement. The greater behavioral recovery in the presence of a transplant was accompanied by anatomical reorganization resulting in greater supraspinal input to spinal cord levels caudal to the lesion (Diener and Bregman, 1998).

\section{DISCUSSION}

Although the recovery of skilled forelimb movement has been examined in the adult rat after various injuries, including spinal cord contusion (Schrimsher and Reier, 1992, 1993), cortical ablations (Castro, 1972a,b; Whishaw and Kolb, 1988; Jones and Shallert, 1992, 1994), striatal or nigrostriatal lesions (Whishaw et al., 1986; Miklyaeva et al., 1994), and globus pallidus lesions (Schneider and Olazabal, 1984), this is the first study to explore the development of forelimb reaching and postural adjustments after high spinal injury in the neonatal rat. We find that transplants of fetal spinal cord tissue mediate recovery of skilled forelimb movement. We suggest that this recovery is mediated by the anatomical reorganization elicited by the transplants. In lesion-only animals, development of postural and limb reflexes is significantly delayed, skilled forelimb function fails to develop, and significant compensatory motor behaviors substitute for the lack of goal-directed reaching and mature postural reactions. After cervical spinal cord overhemisection and transplantation, however, the neonatal rat develops (1) postural and limb reflexes in a time frame similar to normals and (2) skilled forelimb use and accompanying postural adjustments. The significant recovery of forelimb function in the transplant group is supported by dramatic remodeling of supraspinal, segmental and intersegmental projections and their respective interactions with other neurons (Diener and Bregman, 1998). It is likely that the transplant serves both directly as a bridge (conveying supraspinal input to forelimb spinal levels) and as a relay through neurons contained within the transplants (Bregman et al., 1993; Bregman, 1994). The consistency observed in the behavior of each group suggests that common mechanisms unique to each particular group underlie the development. For example, the characteristic behavioral compensations observed by all lesion-only animals may be attributed to common abnormal remodeling of pathways. Although the forelimb skill development of the transplant group is qualitatively different from that of normal animals, we suggest that the differences reflect the sparing of particular components of the normal pattern of connections. The noted behavioral deficiencies were primarily related to the lack of fine details of the movement rather than to either a global absence of the reaching movement per se or an incorporation of abnormal compensatory strategies as observed by lesion-only animals. Sparing of specific skilled forelimb movements was apparent in the presence of a transplant, although there were still deficits in skilled use as compared with normal animals. Transplant animals were significantly better at behavioral tasks than lesion-only animals, indicating that the forelimb development after neonatal cervical spinal cord injury may be a transplant-mediated response.

\section{Complex skill development recovers differently from rhythmical movements}

Activities that involve patterned movement such as locomotion may not be affected as dramatically as those movements that are not initiated by pattern generators. Locomotion is a rhythmical movement guided by local intrinsically activated circuits in the spinal cord and modulated by supraspinal, segmental, and intersegmental input (Grillner, 1975, 1976; Andersson et al., 1978). The current study demonstrates that lesion-only rats use all four limbs to walk overground but are unable to clear obstacles in their path (Bregman et al., 1997; our unpublished results). In fact, lesion-only animals fail to develop forelimb use for any activities (i.e., limb reflex responses and skilled movement) in which muscle action is not governed by central pattern generators. Recovery of input to pathways for nonrhythmical movements may differ from those required for spinal circuits generating rhythmical alternating movements. Lawrence and Kuypers (1968b) reported that deficits in individual forelimb use (such as those apparent in chin placing) were masked when the animal was involved in activities related to forward progression. Alternatively, greater recovery of locomotion compared with reaching may simply indicate that the intrinsic activity of the pattern generators requires less descend- 

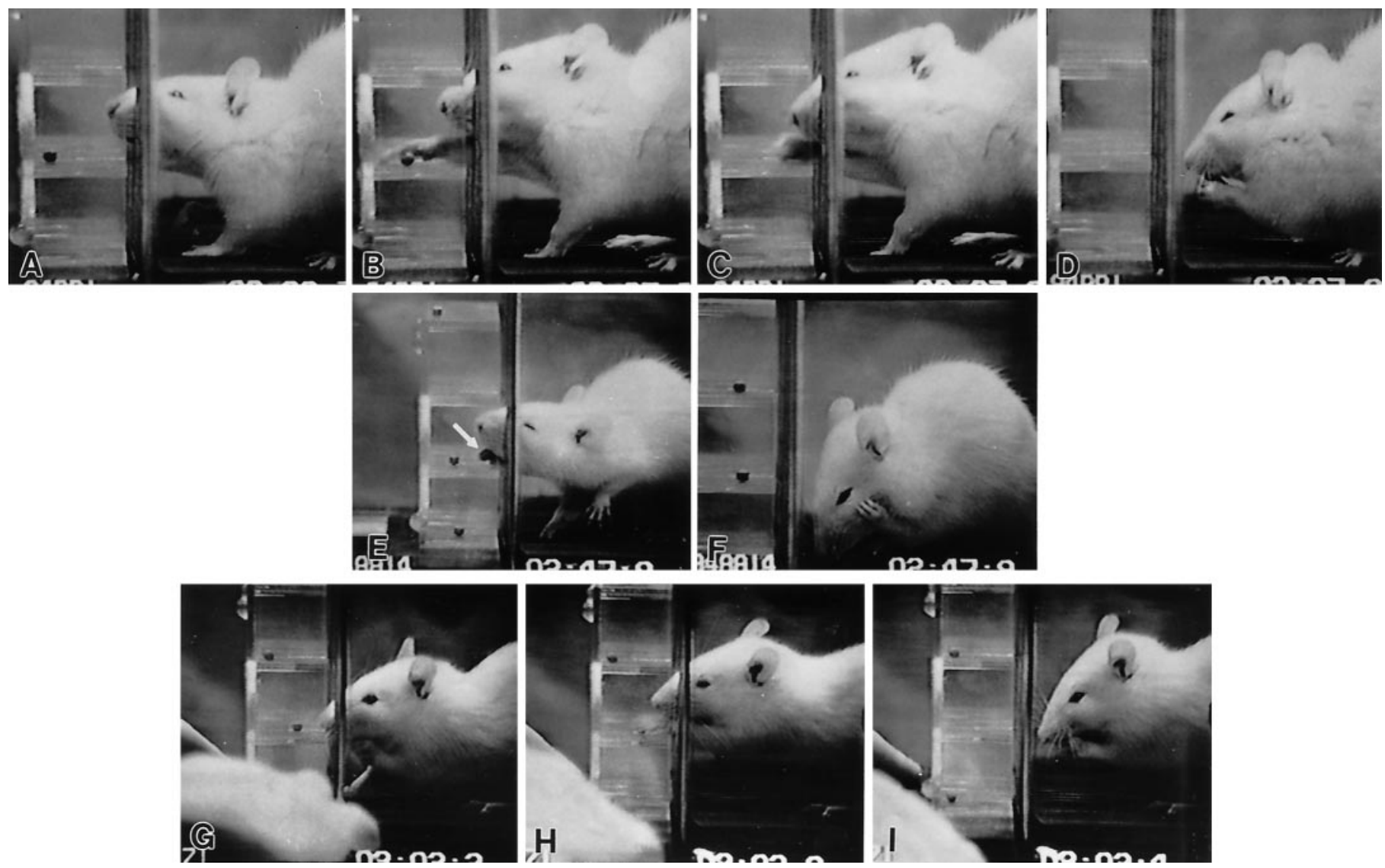

Figure 9. Motor sequences used to reach targets away from the body. $A-D$ (serial images), Normal rats sniff at the target $(A)$, transfer body weight to free a forelimb to reach into a shelf $(B)$, and grasp a pellet $(C)$. After grasping the pellet, the limb withdraws, and the rats sit back on their haunches, bringing both forelimbs to midline to eat the pellet $(D)$. $E, F$ (serial images), Hemisected rats substitute for failed development of target reaching by using their tongues (arrow) to obtain a pellet from the shelf $(E)$. After obtaining the pellet $(E)$, they fail to bring their paws to midline to assist in holding and eating the pellet $(F)$. $G-I$ (serial images), Hemisected plus transplant rats sniff at the pellet and initiate the weight shift to free the reaching forelimb $(G)$. They reach through the grate for the pellet but use a qualitatively different pattern of forelimb and digit extension $(H)$ as compared with normal rats. Transplantation reestablishes postural support, reaching, and bilateral forelimb movements to midline to assist with eating the retrieved pellet ( $I)$.

ing input compared with the more complex neuronal circuitry required for skilled forelimb movements. A complex network of neurons is necessary to execute skilled forelimb use and accompanying postural adjustments (Schneider and Olazabal, 1984; Alstermark et al., 1991a; Miklyaeva et al., 1994) in preparation for limb movement away from the body and for maintenance of balance during limb movement. We suggest that after neonatal cervical spinal cord overhemisection, reduced supraspinal input to neurons in spinal cord segments caudal to the injury and reduced proprioceptive input (Diener and Bregman, 1995, 1998; P. S. Diener, unpublished data) may contribute to the inability to shift body weight to prepare for stepping over obstacles or to raise the limb (Lawrence and Kuypers, 1968b; Alstermark et al., 1984c, 1987a; 1991a). In the current study, transplant animals anticipate and step over the obstacle with both forelimbs, using the left better than the right. Perhaps limb elevation and anticipatory postural reactions occur because of substantial recovery of supraspinal input to the cord, whereas insufficient obstacle clearance results from impaired proprioceptive feedback.

\section{Acquisition of forelimb skills}

Neonatal cervical spinal cord lesion prevents the development of skilled forelimb use in reaching toward or away from the body. Transplant rats, in contrast, develop reaching, grasping, and postural control, although the quality (i.e., manner and frequency) of

\section{PERCENTAGE OF ANIMALS USING THE FORELIMB FOR TARGET REACHING}

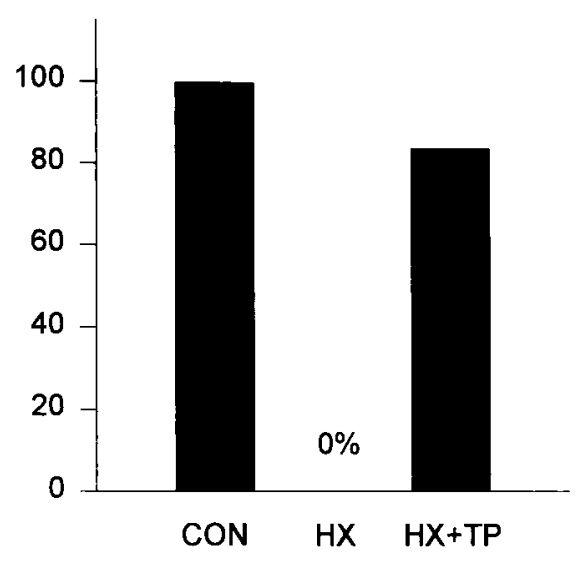

Figure 10. Effect of neonatal cervical spinal cord injury on target reaching. $C O N$ rats $(n=4)$ consistently use either forelimb to retrieve food pellets from horizontal shelves and never engage in compensatory movements. Conversely, $H X$ rats $(n=5)$ consistently failed to develop both reaching and coordinated lower body responses and compensated by using tongue protraction. $H X+T P$ rats $(n=6)$ use a combination of patterns, but more consistently use forelimbs to grasp a pellet. 
A

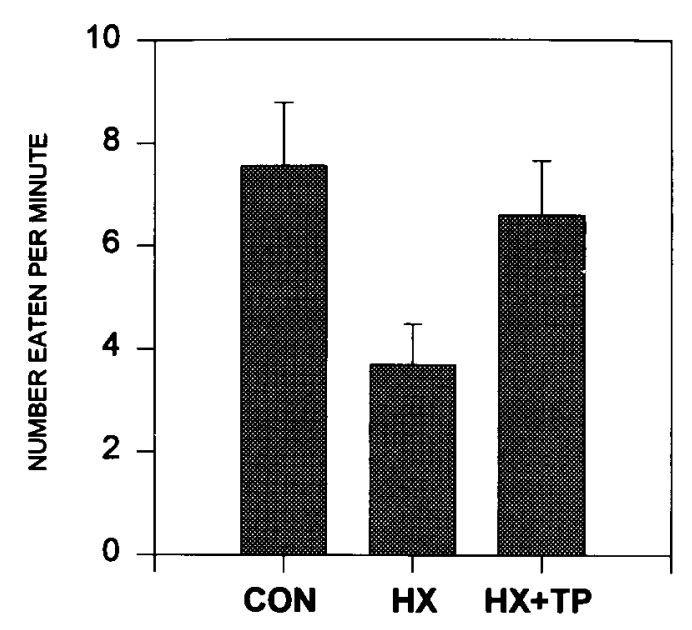

B

REACHING ATTEMPTS

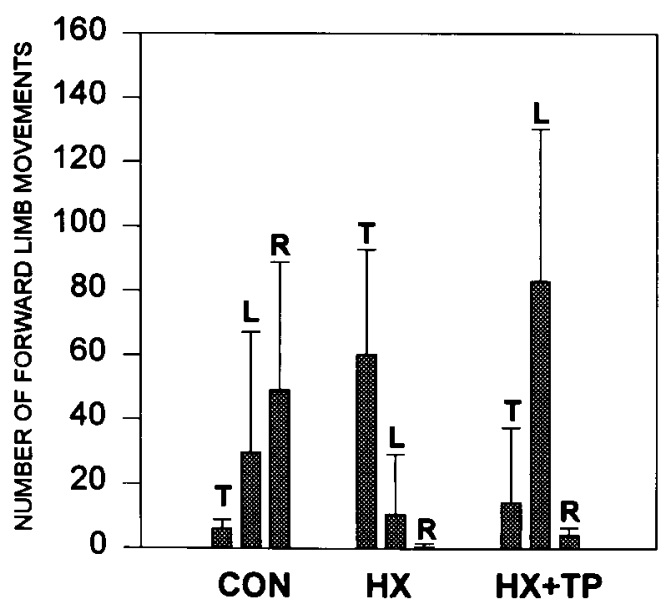

Figure 11. Effect of cervical spinal cord injury on pellet consumption and associated postural adjustments. $A$, Each group score is based on the preferred method of each individual animal for pellet retrieval. The score represents the mean number $( \pm \mathrm{SD})$ of pellets each rat consumed per minute. $H X$ rats ate significantly fewer pellets than both $C O N$ and $H X+T P$ rats, which indicates obvious deficits in motor control for eating. $B$, Both CON and $\mathrm{HX}+\mathrm{TP}$ rats typically use their forepaws to grasp pellets, whereas lesion-only rats primarily use their tongues. When the results shown in $A$ and $B$ are combined, it appears that the transplant rats must reach more frequently to consume the same number of pellets as normal rats. In other words, because of aiming deficits in the transplant animals, they must reach repetitively before actually contacting the pellet. $T$, Tongue; $L$, left forepaw; $R$, right forepaw.

the movement differs from that of normal rats. For example, shoulder joint range of motion is reduced slightly, and adduction of the forelimb is slow to develop. By the time they are weaned, transplant rats use both forelimbs for goal-directed activities, although often without the discreet movement patterns exhibited by normal rats.

We suggest that the effects of transplantation on anatomical remodeling (Diener and Bregman, 1998) lead to the development of the skilled motor behaviors. Corticospinal, brainstem-spinal, and segmental projections influence specific forelimb movements. Goal-directed movements executed by transplant animals can be

\section{POSTURAL ADJUSTMENTS}

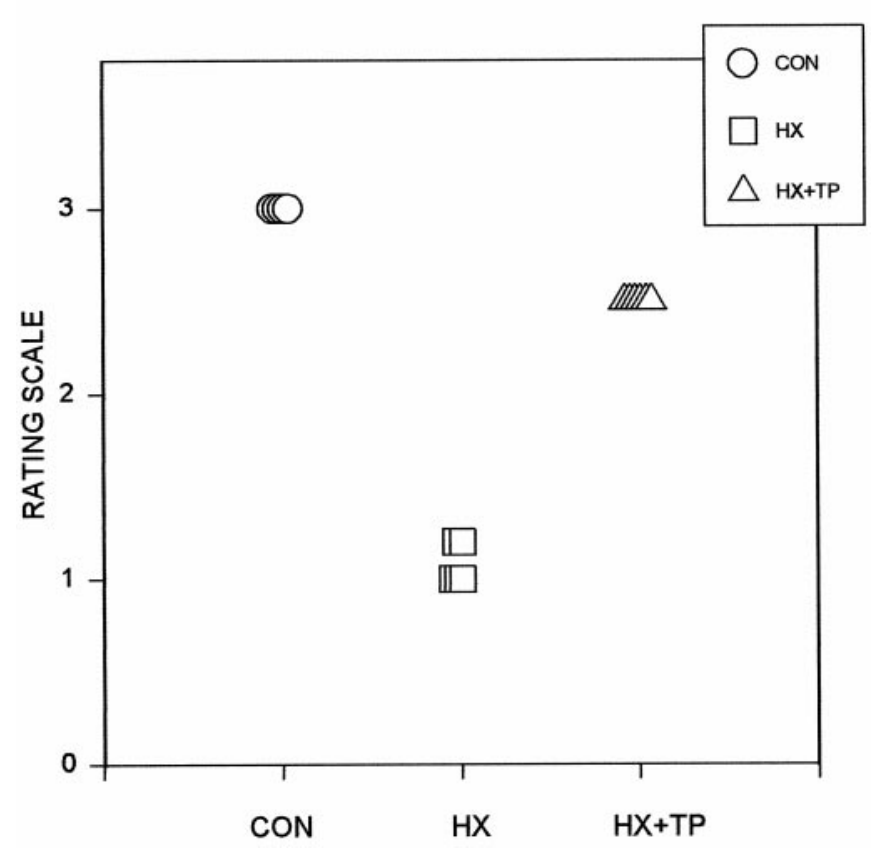

Figure 12. Comparison of the use of postural adjustments in concert with pellet retrieval. Rating scale ranges from 1 to $3: 1$ indicates abnormal behavior and 3 indicates mature, normal movements. Each symbol represents an individual animal in the specified group. $C O N$ rats consistently demonstrate postural adjustments while reaching and eating. $H X$ rats do not reach and therefore fail to make subsequent postural adjustments. They also lack appropriate postural adjustments to maintain balance while retrieving pellets with their tongue. Impaired motor control in each HX animal frequently results in a loss of balance when they move away from their center of gravity. $H X+T P$ rats usually adjust their posture to maintain balance when reaching forward for a food pellet. This represents marked improvements as compared with HX animals in the ability to coordinate postural responses with forelimb use.

distinguished from those of normals in their reaction to movement inaccuracies. When their aim is poor, normal rats quickly correct and modify the movement. For example, when imprecise reaching results in failed grasping, normal rats flex and extend their digits to move the pellet into the paw. Deficits in grasping and adapting movements of the digits to grip objects slightly out of reach result from pyramidal tract lesions (Castro, 1972b) and specifically the pyramidal projection to motoneurons or dendrites of motoneurons (Valverde, 1966; Buxton and Goodman, 1967; Lawrence and Kuypers, 1968a,b, 1972; Illert et al., 1976a,b). Fine adjustments are absent in transplant rats; instead, they repetitively reexecute the entire reach until they touch the target (i.e., batting). Correlations between reduced corticospinal projections into the cord and poor distal control are well supported by other studies suggesting a role for this pathway in detail-oriented movements (Lawrence and Kuypers, 1968a; Castro, 1972b; Reh and Kalil, 1982; Yu, 1984; Schrimsher and Reier, 1992, 1993; Bortoff and Strick, 1993). Batting also has been observed in mature rats after damage to the CNS (Schneider and Olazabal, 1984; Xu and Martin, 1990; Schrimsher and Reier, 1992; Miklyaeva et al., 1994) and may be attributed to reduced proprioceptive feedback (Schneider and Olazabal, 1984; Schrimsher and Reier, 1992, 1993) or altered feedback to propriospinal neurons guiding reaching. The transplant rats in the current study frequently brought 
empty paws to their mouths as if unaware that the pellet had slipped out on the approach. This suggests decreased sensitivity to proprioceptive cues. Paw-checking behavior, as described previously (Schrimsher and Reier, 1992), suggests failed growth of injured primary afferents (Lahr and Stelzner, 1990; Diener and Bregman, 1995); consequently, proprioceptive information cannot be interpreted.

\section{Acquisition of postural control during reaching}

Balance reactions develop in transplant rats, although maturation of postural reflexes, compared with that of normals, is delayed [similar to those in previous reports (Bregman et al., 1993; Bregman, 1994 for review)]. The time necessary for neuronal tracts to both grow from the point of severance and form new connections with spinal neurons may account for this developmental delay. Even in the uninjured CNS, axons grow into the cord at different times and at different rates, often with a waiting period, before arborizing at the target (Schreyer and Jones, 1982; Cabana and Martin, 1985; Joosten et al., 1989). Developmental postural reactions elicited in the majority of transplant animals occur earlier and with better quality of movement compared with lesion-only rats. This suggests that the neuronal rewiring after early injury differs between the two experimental groups.

Postural control and reaching are tightly coordinated in the normal rat so that fluid motion is produced. Postural reactions occur in preparation for, at the initiation of, during, and at the conclusion of the commanded movement (Alstermark and Wessberg, 1985). The inability to make postural adjustments to shift the weight of the body to reach causes lesion-only animals to lose their balance and fall over. The body posture used by transplant rats when reaching resembles that described for normal animals in other studies (Schneider and Olazabal, 1984; Grillner, 1986) as well as that of the control group in the current study. The reestablishment of the disrupted connections in the presence of a transplant spares the development of precise forelimb movements and postural control. Because the patterns of movement differ between lesion-only and transplant rats, we suggest that a different mechanism is being used to generate the executed behaviors. The fetal transplant supports the development of anticipatory movements, associated postural reactions, and goal-directed forelimb skills. Previous studies from this laboratory using a midthoracic spinal cord paradigm indicate that the transplant supports the survival and growth of descending cortical and brainstem spinal axons (Bregman and Reier, 1986; Bregman, 1987b; Bregman et al., 1989, 1991; Bregman and Bernstein-Goral, 1991; Bernstein-Goral and Bregman, 1993) into the transplant and host caudal cord. Other research indicates that supraspinal pathways influence short (C3C4) and long propriospinal and other spinal neurons (Alstermark et al., 1981, 1984a,b, 1987b, 1991b; Alstermark and Sasaki, 1985; Alstermark and Kummel, 1986; Bolton and Tracey, 1992; Wictorin et al., 1992). The transplant is in a position in the animals in the current study to facilitate growth of axons to the spinal cord. Specific influence on propriospinal, forelimb, and hindlimb motor neurons would provide a potential mechanism for improved forelimb use in the transplant as compared with lesion-only animals. Reestablished cortical and brainstem-spinal pathways in the transplant group may correlate with the acquisition of forelimb skills and associated postural adjustments. For example, descending and segmental pathways may (1) reach long propriospinal neurons and spinal neurons to instruct axial and lower body postural muscles about proper reactions to changes in head and upper body position and (2) command short propriospinal neurons, intrinsic cells in the transplant, and brachial motoneurons to produce target reaching (Illert et al., 1976a,b; Alstermark et al., 1981, 1990, 1991a,b; Alstermark and Kummel, 1986). Lack of renewed input results in abnormal behavior as commonly portrayed by lesion-only animals.

\section{Conclusion}

The current study reports several novel findings and provides suggestions for mechanisms that may explain the behavior. After neonatal cervical spinal cord injury, all rats demonstrate delays in development of reflexes and impaired postural reactions and failed acquisition of forelimb skilled use. Abnormal compensatory strategies substitute for these deficiencies. In contrast, all rats with neonatal cervical spinal cord injury plus transplant develop reflex responses, skilled forelimb movements, and postural reactions that are more commensurate with those of normal animals. A common mechanism of action presumably underlies the unique motor performance of each group. Established axonal projections and potential synaptic connections in the transplant group may closely resemble that of the normal group (Diener and Bregman, 1998).

\section{REFERENCES}

Alstermark B, Kummel H (1986) Transneuronal labelling of neurones projecting to forelimb motoneurones in cats performing different movements. Brain Res 376:387-391.

Alstermark B, Sasaki S (1985) Integration in descending motor pathways controlling the forelimb in the cat. 13. Corticospinal effects in shoulder: elbow, wrist, and digit motoneurones. Exp Brain Res 59:353-364.

Alstermark B, Wessberg J (1985) Timing of postural adjustment in relation to forelimb target-reaching in cats. Acta Physiol Scand 125:337-340.

Alstermark B, Lundberg A, Norrsell U, Sybirska E (1981) Integration in descending motor pathways controlling the forelimb in the cat. 9 . Differential behavioural defects after spinal cord lesions interrupting defined pathways from higher centres to motoneurones. Exp Brain Res 42:299-318.

Alstermark B, Lundberg A, Sasaki S (1984a) Integration in descending motor pathways controlling the forelimb in the cat. 10. Inhibitory pathways to forelimb motoneurones via $\mathrm{C} 3-\mathrm{C} 4$ propriospinal neurones. Exp Brain Res 56:279-292.

Alstermark B, Lundberg A, Sasaki S (1984b) Integration in descending motor pathways controlling the forelimb in the cat. 11. Inhibitory pathways from higher motor centres and forelimb afferents to $\mathrm{C} 3-\mathrm{C} 4$ propriospinal neurones. Exp Brain Res 56:293-307.

Alstermark B, Lundberg A, Sasaki S (1984c) Integration in descending motor pathways controlling forelimb in the cat. 12. Interneurones which may mediate descending feed-forward inhibition and feed-back inhibition from the forelimb to $\mathrm{C} 3-\mathrm{C} 4$ propriospinal neurones. Exp Brain Res 56:308-322.

Alstermark B, Gorska T, Lundberg A, Pettersson L-G, Walkowska M (1987a) Effect of different spinal cord lesions on visually guided switching of target-reaching in cats. Neurosci Res 5:63-67.

Alstermark B, Lundberg A, Pinter M, Sasaki S (1987b) Long C3-C5 propriospinal neurones in the cat. Brain Res 404:382-388.

Alstermark B, Lundberg A, Pinter M, Sasaki S (1987c) Subpopulations and functions of long $\mathrm{C} 3-\mathrm{C} 5$ propriospinal neurones. Brain Res 404:395-400.

Alstermark B, Kummel H, Pinter MJ, Tantisira B (1990) Integration in descending motor pathways controlling the forelimb in the cat. 17. Axonal projection and termination of $\mathrm{C} 3-\mathrm{C} 4$ propriospinal neurones in the C6-T1 segments. Exp Brain Res 81:447-461.

Alstermark B, Isa T, Tantisira B (1991a) Integration in descending motor pathways controlling the forelimb in the cat. 18. Morphology: axonal projection and termination of collaterals from C3-C4 propriospinal neurones in the segment of origin. Exp Brain Res 84:561-568.

Alstermark B, Isa T, Tantisira B (1991b) Pyramidal excitation in long propriospinal neurones in the cervical segments of the cat. Exp Brain Res 84:569-582.

Andersson O, Grillner S, Lindquist M, Zomlefer M (1978) Peripheral control of the spinal pattern generators for locomotion in cat. Brain Res 150:625-630.

Bernstein-Goral H, Bregman BS (1993) Spinal cord transplants support 
the regeneration of axotomized neurons after spinal cord lesions at birth: a quantitative double-labeling study. Exp Neurol 123:118-132.

Bolton PS, Tracey DJ (1992) Spinothalamic and propriospinal neurones in the upper cervical spinal cord of the rat: terminations of primary afferent fibers on soma and primary dendrites. Exp Brain Res 92:59-68.

Bortoff GA, Strick PL (1993) Corticospinal terminations in two newworld primates: further evidence that corticomotoneuronal connections provide part of the neural substrate for manual dexterity. J Neurosci 13:5101-5118.

Bregman BS (1987a) Development of serotonin immunoreactivity in the rat spinal cord and its plasticity after neonatal spinal cord lesions. Brain Res 431:245-263.

Bregman BS (1987b) Spinal cord transplants permit the growth or serotonergic axons across the site of neonatal spinal cord transection. Dev Brain Res 34:265-279.

Bregman BS (1994) Recovery of function after spinal cord injury: transplantation strategies. In: Functional neural transplantation (Dunnett SB, Bjorkland A, eds), pp 489-529. New York: Raven.

Bregman BS, Bernstein-Goral H (1991) Both regenerating and latedeveloping pathways contribute to transplant-induced anatomical plasticity after spinal cord lesions at birth. Exp Neurol 112:49-63.

Bregman BS, Goldberger ME (1983) Infant lesion effect: I. Development of motor behavior following neonatal spinal cord damage in cats. Dev Brain Res 9:103-117.

Bregman BS, McAtee M (1993) Embryonic CNS tissue transplantation for studies of development and regeneration. Neuroprotocols 3:17-27.

Bregman BS, Reier PJ (1986) Neural tissue transplants rescue axotomized rubrospinal cells from retrograde death. J Comp Neurol 244:86-95.

Bregman BS, Kunkel-Bagden E, McAtee M, O’Neill A (1989) Extension of the critical period for developmental plasticity of the corticospinal pathway. J Comp Neurol 282:355-370.

Bregman BS, Bernstein-Goral H, Kunkel-Bagden E (1991) CNS transplants promote anatomical plasticity and recovery of function after spinal cord injury. Restor Neurol Neurosci 2:327-338.

Bregman BS, Kunkel-Bagden E, Reier PJ, Dai HN, McAtee M, Gao D (1993) Recovery of function after spinal cord injury: mechanisms underlying transplant-mediated recovery of function differ after spinal cord injury in newborn and adult rats. Exp Neurol 123:3-16.

Bregman BS, Diener PS, McAtee M, Dai HN, James C (1997) Intervention strategies to enhance anatomical plasticity and recovery of function after spinal cord injury. In: Advances in neurology, Vol 72: Neuronal regeneration, reorganization and repair (Seil FJ, ed), pp 257-275. Philadelphia: Lippincott-Raven.

Buxton DF, Goodman DC (1967) Motor function and the corticospinal tracts in the dog and raccoon. J Comp Neurol 129:341-360.

Cabana T, Martin GF (1985) Corticospinal development in the north American opossum: evidence for a sequence in the growth of cortical axons in the spinal cord and for transient projections. Exp Brain Res 23:69-80.

Castro AJ (1972a) Motor performance in rats: the effects of pyramidal tract section. Brain Res 44:313-323.

Castro AJ (1972b) The effects of cortical ablations on digital usage in the rat. Brain Res 37:173-185.

Diener PS, Bregman BS (1994) Neurotrophic factors prevent the death of CNS neurons after spinal cord lesions in newborn rats. NeuroReport 5:1913-1917.

Diener PS, Bregman BS (1995) Fetal spinal cord transplants mediate anatomical reorganization and support the development of target reaching and coordinated postural adjustments after cervical spinal cord injury in neonatal rats. Soc Neurosci Abstr 21:1309.

Diener PS, Bregman BS (1998) Fetal spinal cord transplants support growth of supraspinal and segmental projections after cervical spinal cord hemisection in the neonatal rat. J Neurosci 18:000-000.

Drew T (1991) Visuomotor coordination in locomotion. Curr Opin Neurobiol 1:652-657.

Grillner S (1973) Locomotion in the spinal cat. In: Control of posture and locomotion (Stein RB, Pearson KG, Smith RS, Redford JB, eds), pp 515-535. New York: Plenum.

Grillner S (1975) Locomotion in vertebrates: central mechanisms and reflex interaction. Physiol Rev 55:247-304.

Grillner S (1976) Some aspects of the descending control of the spinal circuits generating locomotor movements. In: Neural control of locomotion (Herman RM, Grillner S, Stein PSG, Stuart DG, eds), pp 351-373. New York: Plenum.
Grillner S (1986) Locomotion in spinal vertebrates: physiology and pharmacology. In: Development and plasticity of the mammalian spinal cord (Goldberger ME, Gorio A, Murray M, eds), pp 311-321. Padova, Italy: Liviana.

Howland DR, Bregman BS, Tessler A, Goldberger ME (1995) Transplants enhance locomotion in neonatal kittens whose spinal cords are transected: a behavioral and anatomical study. Exp Neurol 135:123-145.

Illert M, Lundberg A, Tanaka R (1976a) Integration in descending motor pathways controlling the forelimb in the cat. 1. Pyramidal effects on motoneurones. Exp Brain Res 26:509-519.

Illert M, Lundberg A, Tanaka R (1976b) Integration in descending motor pathways controlling the forelimb in the cat. 2. Convergence on neurones mediating disynaptic cortico-motoneuronal excitation. Exp Brain Res 26:521-540.

Jones TA, Shallert T (1992) Overgrowth and pruning of dendrites in adult rats recovering from neocortical damage. Brain Res 581:156-160.

Jones TA, Shallert T (1994) Use-dependent growth of pyramidal neurons after neocortical damage. J Neurosci 14:2140-2152.

Joosten EAJ, Gribnau AAM, Dederen PJWC (1989) Postnatal development of the corticospinal tract in the rat: an ultrastructural anterograde HRP study. Anat Embryol 179:449-456.

Kunkel-Bagden E, Bregman BS (1990) Spinal cord transplants enhance the recovery of locomotor function after spinal cord injury at birth. Exp Brain Res 81:25-34.

Kunkel-Bagden E, Dai H-N, Bregman BS (1992) Recovery of function after spinal cord hemisection in newborn and adult rats: differential effects on reflex and locomotor function. Exp Neurol 116:40-51.

Lahr SP, Stelzner DJ (1990) Anatomical studies of dorsal column axons and dorsal root ganglion cells after spinal cord injury in the newborn rat. J Comp Neurol 293:377-398.

Lawrence DG, Kuypers HGJM (1968a) The functional organization of the motor system in the monkey. I. The effects of bilateral pyramidal lesions. Brain 91:1-14.

Lawrence DG, Kuypers HGJM (1968b) The functional organization of the motor system in the monkey. II. The effects of lesions of the descending brain stem pathways. Brain 91:15-36.

Lawrence DG, Kuypers HGJM (1972) Developmental aspects of pyramidal motor control in the rhesus monkey. Brain Res 40:117-118.

Miklyaeva EI, Castaneda E, Whishaw IQ (1994) Skilled reaching deficits in unilateral dopamine-depleted rats: impairments in movement and posture and compensatory adjustments. J Neurosci 14:7148-7158.

Reh T, Kalil K (1982) Functional role of regrowing pyramidal tract fibers. J Comp Neurol 211:276-283.

Reier PJ, Bregman BS, Wujek JR (1986) Intraspinal transplantation of embryonic spinal cord tissue in neonatal and adult rats. J Comp Neurol 247:275-296.

Schneider JS, Olazabal UE (1984) Behaviorally specific limb use deficits following globus pallidus lesions in rats. Brain Res 308:341-346.

Schreyer DJ, Jones EG (1982) Growth and target finding by axons of the corticospinal tract in prenatal and postnatal rats. Neuroscience 7:1837-1853.

Schrimsher GW, Reier PJ (1992) Forelimb motor performance following cervical spinal cord contusion injury in the rat. Exp Neurol 117:287-298.

Schrimsher GW, Reier PJ (1993) Forelimb motor performance following dorsal column, dorsolateral funiculi, or ventrolateral funiculi lesions of the cervical spinal cord in the rat. Exp Neurol 120:264-276.

Valverde F (1966) The pyramidal tract in rodents. A study of its relations with the posterior column nuclei, dorsolateral reticular formation of the medulla oblongata, and cervical spinal cord. Z Zellforsch 71:297-363.

Whishaw IQ, Kolb B (1988) Sparing of skilled forelimb reaching and corticospinal projections after neonatal motor cortex removal or hemidecortication in the rat: support for the Kennard doctrine. Brain Res 451:97-114.

Whishaw IQ, O'Connor WT, Dunnett SB (1986) The contributions of motor cortex, nigrostriatal dopamine and caudate-putamen to skilled forelimb use in the rat. Brain 109:805-843.

Wictorin K, Brundin P, Sauer H, Lindvall O, Bjorklund A (1992) Long distance-directed axonal growth from human dopaminergic mesencephalic neuroblasts implanted along the nigrostriatal pathway in 6-hydroxydopamine lesioned adult rats. J Comp Neurol 322:1-20.

Xu XM, Martin GF (1990) The response of rubrospinal neurons to axotomy in the adult opossum, Didelphis Virginiana. Exp Neurol 108:46-54.

Yu J (1984) Reorganization of cerebral control of tactile placing after interrupting a spinal ascending system in cats with pyramid section. Brain Res 290:19-23. 